\title{
Do terrorists make a difference in criminal networks? An empirical analysis on illicit drug and narco-terror networks in their prioritization between security and efficiency
}

\author{
Mustafa Coşar Ünal \\ Department of Political Science and Public Administration, Bilkent University, Ankara, Turkey
}

\section{A R T I C L E I N F O}

\section{Keywords:}

Dark/covert networks

Security-efficiency tradeoff

Narcoterrorism

Social network analysis

\begin{abstract}
A B S T R A C T
By using Social Network Analysis (SNA), this study empirically analyzes five PKK Affiliated narco-terror and five illicit drug networks in the Turkish context to identify and compare their approach to the security-efficiency tradeoff. Results revealed that although terrorist members are in key roles and powerful positions (central and intermediary), average scores of cohesion and centrality of narco-terror networks seem to be only slightly more security driven than those of illicit drug networks. However, analyses of individual networks from both types yield no clear structural distinction in prioritizing between security and efficiency. This study, in general, finds that networks from both camps are structurally more efficiency driven. They are denser with more direct ties; generally clustered into sub-groups attached to networks' cores and peripheries; they reflect coreness, where key players act in pivotal positions with high power, centrality, and brokerage to efficiently control and coordinate network activities. This is then found to cause security vulnerabilities of greater visibility (susceptibility of disruption) and high dependence on central actors constituting structural holes (possible elimination of entire network when compromised). However, sample networks strive to balance the security by keeping the paths-for the flow of information-shorter and being clustered into denser sub-groups with strong pre-existing trust-ties (kinship and friendship) which reduces the risk of detection and infiltration. This study argues that the nature of objective dominates the structural characteristics regarding the security-efficiency dilemma, and thus, both illicit drug and PKK affiliated narco-terror networks in Turkish context are driven by material incentives relying more on efficiency and striving to balance organizational security with social characteristics (trust ties).
\end{abstract}

\section{Introduction}

Terrorist and criminal networks face an enduring dilemma; to survive they have to maintain security by staying secret, and to accomplish their aims and objectives they have to ensure efficient communication and coordination through flow of information and resources. Known as the security-efficiency tradeoff, the interplay between secrecy and action is considered vital in how covert networks are structured and operate (Morselli et al., 2007; Crossley et al., 2012; Lindelauf et al., 2009; Baker and Faulkner, 1993; Erickson, 1981; Milward and Raab, 2006; Krebs, 2002). The nature of the activity (e.g., aim, objective, motive), whether terrorist or criminal, is considered to create particular difference in these networks' prioritizing between security/secrecy and efficiency (Morselli et al., 2007). However, existing literature contains limited empirical studies based on observed data on covert networks in general (e.g., see Koschade, 2006; Belli et al., 2015; Krebs, 2002; Natarajan,
2000, 2006; McGloin and Kirk, 2010; Klerks, 2001; Edwards, 2014; Bright et al., 2012; Jordan et al., 2008) and on covert networks' behavior regarding the security-efficiency dilemma in particular (e.g., see Morselli et al., 2007; Crossley et al., 2012; Enders and Su, 2007; Baker and Faulkner, 1993; Erickson, 1981; Helfstein and Wright, 2011).

This study empirically tests the main assertions of the security-efficiency trade-off in two ways. First, this study explores the structural characteristics of sampled covert networks from Turkish context, narcoterror and illicit drug networks, to identify their security-efficiency trade-off in general. Second, given that terrorist networks are argued to rely more on security (Morselli et al., 2007), this study identifies whether or not differences exist between narco-terror networks, where drug trafficking acts intermediately to finance terrorist activities, and illicit drug networks in prioritizing between security and efficiency in particular. $^{1}$

By applying Social Network Analysis (SNA) to observed data

\footnotetext{
E-mail address: cosar.unal@bilkent.edu.tr.

${ }^{1}$ This study is continuation of a research in which only illicit drug networks are analyzed in their prioritizing between security and efficiency.
} 
extracted from official government records, this study examines structural characteristics of 10 randomly selected sample cases: five illicit drug networks (DGN) and five narco-terror networks (NTN) that operated in Turkey and its related regions, i.e., Central Asia, Europe, and the Balkans. The sample cases vary in terms of the trafficking of different drugs, such as heroine, hashish, marijuana, morphine base, and certain synthetic drugs (e.g., captagon, ecstasy). The sampled networks include a total of 145 members, 71 of whom from DGNs and 74 from NTNs. This study resorts to SNA metrics to elucidate network structures (relational patterns) and topographies to identify sample networks' prioritization between security and efficiency. These include measures to identify cohesion and connectedness (e.g., density, average path length, clustering coefficient, transitivity, average nodal degree), organizational make-up (e.g., degree, closeness, betweenness centrality and group centralizations, and core-peripheriness), role and positions of key players and terrorists, and other related structural inference measures.

This research makes a significant contribution to the limited empirical literature by applying an analytical network method to a rich set of observed data. Despite the abundance of studies analyzing covert networks, empirical studies with observed data is very limited due to difficulty in accessing such data (Rene and Hulst, 2009: 110; Helfstein and Wright, 2011: 785). Thus, as Crossley et al. (2012) pointed out, prior research on dark networks, in general, has been based mostly on simulations and theoretical models (e.g. see, Lindelauf et al., 2009; Enders and Su, 2007) and only limited number of studies in the literature have been based on data (e.g., Belli et al., 2015; Crossley et al., 2012; Jordan et al., 2008; Krebs, 2002; Natarajan, 2006; Koschade, 2006; Morselli et al., 2007; Baker and Faulkner, 1993). Everton and Cunningham (2015: 2) acknowledge that many of the studies on security efficiency characterization have been qualitative in nature and lack standard SNA measures. Moreover, as Zech and Gabbay (2016: 215) underline, covert nature of clandestine/dark networks makes them difficult to study, and mapping out their internal structure-comprised of latent relations-makes this problem particularly acute, which reflects a discrepancy between theoretical and empirical SNA studies in the literature. In all these regards, this study stands out analyzing 10 cases by leaning on a rich set of observed data extracted from official government databases which includes multiple sources from different agencies (law enforcement and criminal intelligence units, courts).

Second, there has been no empirical study exploring and comparing structural characteristics and the behavioral nature of illicit drug networks and narco-terror groups in opting for security or efficiency, nor has there been an empirical study on Turkish criminal networks' prioritizing between secrecy/security and efficiency.

This study follows with a brief literature review on the structural characteristics of terrorist and criminal organizations as dark/covert networks with a particular focus on behaviors of these networks in the security-efficiency tradeoff. Second, it introduces a conceptual model through which it develops hypotheses based on earlier empirical accounts and discusses the operationalization of related SNA metrics followed by a discussion on the nature and characteristics of the data. Next, it descriptively analyzes each individual sample networks in terms of their network and actor level structures and topographies. In that, it provides general introductory information on each sample network and engages in individual level node analyses in which key players, their positions, positions of terrorist members (for narco-terror samples), pre-existing trust ties, and certain related network level characteristics are briefly discussed along with certain results related to the general topography (e.g., cohesion, centrality) of individual cases towards their prioritization between security and efficiency. Finally, it addresses each developed hypotheses by discussing the findings among sample networks and, in that, it compares networks social and structural characteristics among sample networks and between other covert networks analyzed in the similar empirical studies in the literature.

\section{Literature review}

There are two interrelated debates in the literature on the structural characteristics of covert networks: one, how covert networks respond to the efficiency security trade-off in general; two, how the nature of activity, terrorist and criminal, makes a difference, if any, in these covert networks' structural characteristics when prioritizing between security and efficiency. Efficiency, is conceptualized as profit for crime groups and political influence or political gains for terrorist and insurgent groups, and security/secrecy, is conceptualized as maintaining network sustainability and resilience, constitute the two most important factors relating to capacity and persistence/survival shaping the structure of covert networks. Given this, do we see structural differences between terrorist and criminal networks where they have similar methods but divergent motivation and end goals?

While both, drug and terror groups are risk averse regarding security, Morselli et al. (2007: 143-5) claim that these groups differ in operational character: While terror groups weigh security over efficiency, drug groups prioritize efficiency over security. This makes them act methodically different. 'Time-to-task' is shorter for drug and longer for terror groups because efficiency driven criminal networks demand quicker returns than ideologically motivated terror groups who are willing to trade some efficiency for security. Given that drug networks are more flexible and pragmatic easily replacing one another (Natarajan, 2006; Milward and Raab, 2006), they tend to opt for efficiency (Morselli et al., 2007: 143; Morselli, 2009). However, Crossley et al. (2012: 3) argue that this might not always the case because efficiency as quick return of material resources is also very important to ideologically motivated networks and the relative importance of secrecy and efficiency may vary by temporality and actor characteristics. Thus, ideologically motivated covert groups view efficiency and secrecy as a compatible aim rather than a trade-off. Concurring with this arguments of Crossley et al. (2012), Everton and Cunningham (2015: 2) point out that covert networks seek to balance operational security and efficiency which culminates in temporally dynamic structure indicating shifting prioritization between security and efficiency in covert networks' operational process and life cycles.

On the other hand, Erickson (1981) reports that long endured dark networks that have established a reputation prefer security over efficiency. Enders and Su (2007: 54) underscore the temporality and dynamic nature of networks' response to this trade-off and argue that terror groups choose to reduce communication when faced with security threats resulting in less coordination and varied attacks. Helfstein and Wright (2011:805-06), claim-by evaluating certain terrorist groups-that attack networks become increasingly dense as they get closer to attack execution implying the dynamic nature and temporal differences in covert networks' response to the trade-off. Concurring temporality and dynamic structure of these networks, Lindelauf et al. (2009) argue that dark networks yield star-like structure in their initial stage in a hostile environment and when passed this stage they reflect small world characteristics. Moreover, Morselli et al. (2007: 152-53) argue that, as opposed to criminal networks, terrorist networks do not reflect core-periphery structures. Raab and Milward (2003: 413-9) and Helfstein and Wright (2011, 805-07) argue that terrorist networks might have cores that facilitate terrorist activities with specialized members for certain technical issues of finance, strategy, and planning.

In the end, results are mixed (Oliver et al., 2014: 13) and the conflicting imperatives of secrecy and efficiency (Morselli et al., 2007; Crossley et al., 2012; Lindelauf et al., 2009; Baker and Faulkner, 1993), persistence and capacity, differentiation and integration (Milward and Raab, 2006), or secrecy and communication (Enders and Su, 2007) are driving forces for a dark network in designing its structure and operation. It should also be noted that there exist certain technical and methodological reasons for the competing arguments on centrality and density of covert networks. As Everton and Cunningham (2015: 2) point out, these mainly include measurement problem, e.g., resorting to a 
single centrality measures; omitting multi-relational nature of dark networks and failing to outline different types of relations-i.e., treating all different relations as a single type; and not accounting for the context and environment in which covert networks operate.

Notwithstanding, certain specific structural characteristics are used to identify dark networks' behavior in organizational security and efficiency. These mainly include network cohesion (density), connectedness, (degree) centrality and group centralization, small worldliness (clustering into tight-knit groups), core-periphery structures (coreness), and role and position of key players, and existence of pre-existing (trust) ties. In that, the general argument is that centralized and dense networks are positively associated with operational efficiency since they are more effective in mobilizing resource and information and thus more efficient in communication and coordination (Baker and Faulkner, 1993; Morselli et al., 2007). Hence, if a dark network weighs security over efficiency, that network is assumed to be sparse (low in density and short in average geodesic distance [average path length]) and degree decentralized, i.e., flattened and horizontally structured (Baker and Faulkner, 1993; Enders and Su, 2007; Klerks, 2001; Krebs, 2002; Morselli et al., 2007; Oliver et al., 2014: 9; Raab and Milward, 2003; Rodriguez, 2009). More specifically, the chance of compromise increases when decisions/information have a longer path in the network. Higher density and clustering into denser sub-groups can decrease risk of compromise, but then higher density causes another security risk with more visibility in action. The alternative is, inevitably, having a centralized hub that coordinates and controls all activities through direct contact (Lindelauf et al., 2009: 136; Crossley et al., 2012: 2-3). However, should the hub/cell/network be compromised, that centralized network can suffer elimination.

As noted by Crossley et al. (2012: 2-3) and Everton and Cunningham (2015: 1-2), there exist competing arguments on covert networks' centralization and cohesion. While some argue that low density and decentralization are more secure with minimal network damage when a single actor is compromised (e.g., Raab and Milward, 2003; Enders and Su, 2007), others suggest that high density or centralization leads to increased in-group solidarity, self-sacrifice and yields more trust and secrecy, thereby reducing the likelihood of compromise, defection, and infiltration (Lindelauf et al., 2009). Baker and Faulkner (1993: 838) support the latter argument by claiming that while decentralization might seem the best for secrecy and efficiency, higher centralization is necessary when accomplishing complex tasks and when a dark network requires secrecy and efficiency simultaneously. Sparrow (1991), on the other hand, claims that high and low centralization can be balanced by having localized dense clusters in a globally sparse networks chain where the clusters bring benefits such as motivation, solidarity, selfsacrifice, easy recruitment and trust via pre-existing ties where the sparse structure allows for loose connections, minimized security risk of infiltration and reduced damage when compromised. Concurring with this, Watts (1999) and Krebs (2002) underline the importance of small dense clusters reflecting small world characteristics in which these clusters, as Granovetter (1993) claim, are tied to one another via 'significant' weak ties indicating the vitality of these loose connections when analyzing dark networks.

However, the dilemma does not end. While loose connections may lead to structural holes and less coordination between clusters (Everton, 2008), less centralized networks are more flexible and responsive and thus they can easily adapt to changing environments (Raab and Milward, 2003; Natarajan, 2006). While increased density and degree centralization leads to greater cluster visibility and therefore susceptibility to detection (Crossley et al., 2012), it brings efficiency in decision making mechanism by greater communication, coordination and more rational flow of information and resources (Everton and Cunningham 2015:2). So, there exists strength and weakness for different structures yielding compensations of density, centralization, path length, and clustering. Hence, as Everton and Cunningham (2015: 2) point out covert networks' effort for balancing security and efficiency leads to temporally dynamic structure in terms of centrality and density. In this regard, covert networks reflect microstructures of clusters and cliques as well as core-periphery structures (Oliver et al. 2012: 10) to balance strengths and weaknesses stemming from different structural characteristics (Crossley et al., 2012: 4).

In the end, as many argue (e.g., Raab and Milward, 2003; Milward and Raab, 2006; Everton and Cunningham 2015; Erickson, 1981; Baker and Faulkner, 1993), networks are hardly static organisms, they are subject to constant change based on shifting circumstantial factors and conditional dynamics, thus, they have a dynamic structure to adapt to the context and environment within which they operate. As Crossley et al. (2012: 3) specifically point out, networks might have multiplex structures in which scores of centrality, centralization, and density may vary where different layers of a network are responsible for different activities and resources (e.g., flow of money, drug, information and so forth). Therefore, covert networks may reflect both relatively more centralized-decentralized and denser-sparser natures as a whole and in their micro structures. This may also change depending on the context, temporality, layer, type of activity/action, objective, member characteristics and related other circumstantial conditions where the overall network or its particular segment/cell/sub-group operate (Oliver et al., 2014: 11; Erickson, 1981: 189; Crossley et al., 2012; Baker and Faulkner, 1993). Morselli (2009: 160) concurs this and points out that criminal activities by covert networks should not be framed within the boundaries of formal organizational structures due to the dynamic nature of structures and participants. Last but not the least, covert networks may have similar methods and divergent ends but their structures, communication skills, activities, secrecy and trust levels are shaped based mainly on the geo-spatial/contextual conditions (Makarenko and Mesquita, 2014; Howard and Traughber, 2008; Asal et al., 2015), where circumstantial contingencies and strategic needs are influential on the structural characteristics of these networks (Raab and Milward, 2003).

In addition to centrality and density arguments, roles and positions of members, particularly the structural position of key players in covert networks reflect another important indication of covert networks' efforts in balancing between security/secrecy and efficiency. Duxbury and Haynie (2018: 239) and Baker and Faulkner (1993: 856) underline that key players (high profile members/distributors) opt to insulate themselves from the core activity and incidences of network activity by connecting only a few actors. Morselli (2010: 390) concurs this and suggests that low (degree) centrality and high betweenness indicate a strategic positioning of key players being in the periphery rather than in the hands-on action of the core. Thus, key players (top executives) are positioned in periphery structures, i.e., with less direct ties and less visibility, to mitigate security vulnerabilities by protecting key actors and limiting the risk of disruption by elimination of key players.

Social characteristics of dark networks, in addition to their structural characteristics, indicate another crucial aspect. In that, the preexisting ties and social bonds known as 'trust ties' constitute an important character in these networks' structures and operation. Raab and Milward (2003) and Milward and Raab (2006: 349) argue that drug and terror groups are both risk averse and this feature has permeated in almost every aspect of their development and operation in which 'ties of trust' plays an important role in their sustainability, resilience and growth. Similarly, Oliver et al. (2014: 4) and Erickson (1981) claim that pre-existing social interaction is believed to reduce the vulnerability of covert networks because these prior ties increase their operational security and thus their resilience. Concurring with this, Crossley et al. (2012: 3) argue that trust ties and social bonds facilitate recruitment and self-sacrifice. This is because, as Erickson (1981) and Sageman (2011) assert, pre-existing contacts are important for the growth of networks making social ties and trust-based relations inevitable. Jordan et al. (2008: 36) specifically argue that the covert networks are more socially restrictive based on pre-existing (familial and friendship) trust ties as they get smaller in size and, thus, the odds for security agencies 
to detect them is mitigated. In support of these arguments, Krebs (2002) in his analysis of terrorist network of September 11 attacks, suggests that, for reactive and proactive preventive measures, 'trust ties' between and among members are to be identified to understand and disrupt a covert/dark network. This is because, as Paoli (2001) points out, most recruits come from the 'ties of trust' which brings the social bond forefront.

Therefore, in addition to the network characteristics, type of relationships among members, their social bonds in functioning and social structure, and their connection with the terrorist groups are considered among important factors to understand the relational structures of covert networks. Social bonds also help to explain the motivation of drug traffickers, for example strong social bonds help an organizational culture that includes strong communication skills and trust; often the result of knowing each other prior to trafficking activity, as a relative or friend. Also, members may bond together because of their ethnic background or tribal relationship, establishing a stronger and resilient network structure to maintain power and position against laws enforcement units and rival networks (Raab and Milward, 2003; Hudson et al., 2002). Most importantly, social characteristics of covert networks that reveal growing along lines of trust and reflect intense pre-existing social ties, which have influence on the structural patterns of these networks. For instance, recruitment through strong familial or tribal ties suggest that the network will become denser which is underlined as formidable triad by Granovetter (1973).

\section{Method and data}

Data

This study uses data extracted and coded from official government records ${ }^{2}$ which is part of a broader dataset extracted for previous research (Turhal, 2015) ${ }^{3}$. It includes 10 randomly sampled networks including five DGNs and five NTNs. The sample cases vary in terms of the trafficking of different drugs, such as heroine, hashish, marijuana, morphine base, and certain synthetic drugs (e.g., captagon, ecstasy). The sampled networks include a total of 145 members ( 71 for DGNs and 74 for NTNs).

Krebs (2002: 51) argue that SNA data should identify two sets of information, "task" and "trust" data, to plot an accurate picture of a covert network. "Task" data includes all logs and records of telecommunications (including interactions via Internet), travels, intelligence data from surveillance and infiltration as well as public and court records. "Trust" data includes prior contacts through family, school, clubs, and other organizations. To have both task and trust information, this study utilizes data collected and coded from several documents archived by different security agencies and entities across the span of investigation and prosecution processes. These include documents from the database of law enforcement units (e.g., summary reports of police investigation and interrogation) and court files (prosecution). The law enforcement unit database contains case summary reports, police surveillance records including physical (i.e., face-to-face meetings, courier-based interaction) and electronic sources (i.e., phone

\footnotetext{
${ }^{2}$ The database of the Turkish Anti-Smuggling and Organized Crime Department (ASOCD). The ASOCD database enables (through a keyword search of "terror" and related terms) to separately extract cases from two pools, based on a distinctive criterion whether or not a drug trafficking case was linked to the PKK (Turhal, 2015). The networks analyzed in this research are sampled from the pool of 100 cases that are sampled from the larger pool of ASOCD database. Five randomly selected drug trafficking cases with no linkage to terrorist organizations (from a pool of 50) and another five randomly selected cases from the PKK-linked pool (of 50), are compiled (in total 10 cases) to compare and contrast the two groups for the purpose of this research.

${ }^{3}$ The author would like to express his gratitude to Dr. Turhal for his generosity in sharing the data.
}

calls, Internet connections), wiretaps (i.e., content analysis), offender statements, court records/proceedings, and other case related documents from the official records. The majority of network data (relational ties) was taken from reports prepared by police surveillance teams during the investigation process and transcripts of wiretaps from police case files. These data were particularly useful in elucidating ingroup relations, key players, and communications flows having included information on latent relations between and among network members (Turhal, 2015). ${ }^{4}$

The network data are coded according to interactional criteria consistent with the SNA method using coding techniques that are described in several resources that include Wasserman and Faust (1994); Freeman et al. (1979), and Hanneman and Riddle (2005). These include the transactional content measure, exploring the exchange of information and resources within the relations between offenders in networks. In order to refrain from any bias that would stem from coding differences (given the use of multiple sources that yield inconsistencies in format, template and manner) the two sample cases of the larger pool of data were coded by two external researchers to conduct inter-rater reliability tests through using the percentage agreement method. The results reflected only insignificant differences in coding the information stored in the extracted case files that indicates no systematic bias stemming from coding process (See Turhal, 2015). ${ }^{5}$

The data include the symmetrical relations (adjacency matrix) based on these interactions reflecting "ties" in a binary network (0-1). There also exist valued data indicating the strength of ties in a 1-3 scale indicating weak, moderate, or strong relationships between offenders (based on frequency and duration of interaction). The valued data is used when plotting sociograms by underlining the tie strength with bolder lines and arrow heads. In the end, calculation of the data reveals a one-mode symmetric (non-directional/undirected) nature.

Conversely, certain data limitations should be noted. As Van der Hulst (2009: 110) argued, compiling complete network data, particularly for dark networks, is very difficult since these networks remain ontologically covert and underground to survive. More importantly, covert/dark networks reflect "incompleteness," indicating that some links within dark networks will be missed; "fuzzy boundaries" in which the borders of any covert network is unclear; and "dynamism" in which they constantly change over time (Sparrow, 1991: 261-263). For the fuzzy boundaries, in particular, the capabilities of SNA in relation to small group networks is critical (Burcher and Whelan, 2015: 104-105). Yet, data used here reflect only static representation of structural characteristics and do not include temporal changes.

Still, as Zech and Gabbay (2016: 215) pointed out, and mapping out internal structures-comprised of latent relations-of covert networks is particularly an acute problem leading to a discrepancy between theoretical and empirical studies. More importantly, as Bright et al. (2012: 153) and Van der Hulst (2009: 110) underlined, having institutional access to law enforcement agencies' databases is crucial in having alternative enquiries on information related to the relational and structural patterns of these networks. The data sample for this study includes a wide array of data sources which enables not just elaboration but also verification of relational data from multiple sources.

\footnotetext{
${ }^{4}$ Offender statements in police investigations and in courts include individual demographic information such as date and place of birth, education level, occupation, marital status, income, nationality, number of children as well as offenders' interactions/communications in networks. However, the majority of the network data related to operational and organizational structure was taken mostly from surveillance reports prepared by surveillance teams during the investigation process and wiretaps from police case files and court documents which were particularly useful since they included information that reflected relations between and among network members.

${ }^{5}$ For those who are interested in details, a separate document that elaborates all procedures and results from inter-rater reliability test is ready to be provided by the author.
} 


\section{Conceptual model}

First, this section identifies the main assertions made in empirical studies on covert networks' behavior regarding the security-efficiency tradeoff. Then, hypotheses are developed based on these main claims by identifying whether or not illicit drug networks (as criminal groups) are any different from narco-terror groups (having terrorist members) and analyzing their structural characteristics/patterns in prioritizing between security and efficiency.

Before going over the hypotheses, it should be noted that narcoterrorism has no standard definition and different conceptualizations refer to different foci and implications (Björnehed, 2004: 306). In this study, narcoterrorism refers to terrorists' use of narcotics trade wherein the narcotic trafficking organization serves as the referent object; the prime activity is the illegal trade of narcotics and terrorist members resort to it for financial gains.

\section{Hypotheses}

Hypothesis 1: The general argument is that covert networks are more concerned with security and therefore tend to be sparser with minimized communication and a more degree decentralized structure (Baker and Faulkner, 1993; Erickson, 1981). Crossley et al. (2012) specifically argue that covert networks become less dense and less degree centralized as they become more secret. Terror activity is more concealed given the level of the risk, magnitude of the destructiveness/ cost, ideological motivation (see e.g., Sageman, 2011; Mayntz, 2004), and as Morselli et al. (2007) argued, longer time-to-task nature of activity, therefore, structural characteristics of terrorist networks are expected to be more security driven.

H1. Illicit drug networks overall are denser and more degree centralized compared to narco-terror networks

Hypothesis 2: Morselli et al. (2007: 151)—by underlining the divergent nature of objectives-argue that criminal networks, particularly illicit drug organizations, are driven by profit and thus reflect more efficient communication indicating more incidence of action in a shorter time frame for expected pay-off while the situation is the opposite for terrorist networks. Thus, despite that security is the principal concern for most criminal networks, shaping a network in favor of increased insulation of offenders is typically a luxury which is more like to be found in terrorist networks because they are not profit oriented (Morselli, 2009: 159). This notion has important influence on how these networks are structured: while terrorist networks are featured with more distance between members, criminal networks are marked with shorter distance reflecting dense clusters and cliques.

H2. Illicit drug networks are more clustered into dense sub-groups and have shorter (path) distance compared to narco-terror networks.

Hypothesis 3: Morselli et al. (2007: 148) also argue that criminal networks, particularly illicit drug groups, are structurally built out from a core to ensure more efficient communication and coordination, while terrorist networks lack a core because of concerns over security. Since the data in this study reflect only static representation, only the coreness and core-periphery structures are analyzed to address the following hypothesis.

H3. Illicit drug networks have more coreness reflecting more coreperiphery structures compared to narco-terror networks.

Hypothesis 4: Baker and Faulkner (1993: 856) argue that criminal groups weighed security over efficiency by having certain key players (top executives) positioned in periphery structures, i.e., with less direct ties and less visibility, to mitigate security vulnerabilities. In that high profile members/distributors insulate themselves from more incidence of network activity by connecting only a few actors. While it decreases efficiency, it protects key actors and limits the risk of disruption by elimination of key players (Duxbury and Haynie, 2018: 239).

Identifying key players' roles and positions in a network is vital to better understanding the relational patterns and structures of covert networks (Sparrow, 1991; Natarajan, 2000, 2006; Van der Hulst, 2009; McGloin and Kirk, 2010). There is no literature on formal role distributions of drug networks (Bright et al., 2012: 162) and roles and labor divisions in covert networks vary according to contextual dynamics of geo-political regions (e.g., type of drug, cycle of trafficking activity [e.g., production, dissemination]) and the cultural environment in which they operate (Levitt and Venkatesh, 2000; Natarajan, 2006; Bright et al., 2012). Given these, despite their small size (average 14.8) sample networks include various roles assigned to different layers. Three recognizable hierarchical layers exist in sample networks: strategic, tactical, and operating. For the purpose of testing this hypothesis, only strategic level roles and terrorist members' positions (for NTN cases) have been identified and discussed.

Strategic level offenders in the sampled drug networks constitute the central command structure. This typically includes organizational leaders (ORL) and subordinate high-level managers (HLM), core actors initiating and controlling trafficking activity. ORLs are crime initiators and final decision makers. HLMs play a brokerage role between other layers, and they plan, design and control trafficking activities (for similar role classifications, see Van der Hulst, 2009; Crossley et al., 2012; Natarajan, 2006; Bright et al., 2012). Leaders and managers are identified by police surveillance reports and offender statements. For the sampled cases, most ORLs are clear, however, they were not identified in certain cases. There is also a drug provider (DP) role which refers to the members who supply the illicit drugs to be trafficked. She/he is mostly tied to strategic level offenders. Thus, the positions of ORLs and HLMs are analyzed as key players, while DPs are only identified.

Terrorist member simply refers to offenders who are strongly linked to the Kurdistan Workers' Party (PKK), ${ }^{6}$ an insurgent group employing intense terrorist activity (Ünal, 2014, 2016). Through four decades of conflict, the PKK has resorted to various types of illicit activities in and outside Turkey including extortion, human smuggling, and other criminal activities (Ünal, 2012, 2016). However, drug trafficking has sustained the greatest part of the PKK's financing activities (e.g., Makarenko and Mesquita, 2014; Howard and Traughber, 2008). Turkey is geographically on a major drug trafficking path called the "Balkan Route." Turkey is, therefore, characterized as a transit country between the drug producing countries of Central Asia and the Middle East and destination countries mostly in Europe (Makarenko and Mesquita, 2014; Howard and Traughber, 2008). Terrorist members are identified based on evidence including prior records, offender statements (confession), and reports from criminal intelligence units. Linkages to the PKK include physical support and involvement of PKK activities, financial support in transferring money to PKK members, and possessing illegal documents related to the PKK. The nodes referring to PKK members are denoted with the prefix " $\mathrm{T}$ " to be easily recognized in sociograms and tables (e.g., T12 or T5-HLM).

H4. Key players in drug networks tend to be positioned more in the networks' core for more efficient control and coordination while key players and terrorists in Narco-terror networks are positioned more in periphery structures to maintain more security.

Hypothesis 5 and 6: Erickson (1981: 189) argues that long endured networks with a reputation tend to opt for security and pre-existing social structure is a significant determinant of covert networks in maintaining organizational security (also see Raab and Milward, 2003; Oliver et al., 2014; Crossley et al., 2012; Krebs, 2002; Everton and

\footnotetext{
${ }^{6}$ The nature of the PKK, whether an insurgency or a terrorist organization is beyond the scope of this study. The PKK is denoted with terrorism since it is recognized as a terrorist organization by many countries and international bodies (see Ünal, 2016).
} 
Cunningham, 2015). Analysis of trust-ties is further elaborated on through kinship and peer relationships. Direct kinship ties (e.g., brother, husband, uncle, cousin), other "blood ties" including indirect kinship ties, such as uncle's brother or nephew's wife, and friendship between offenders are identified from offender statements and corresponding census reports. Since the claim does not make distinction between terrorist and criminal networks, hypotheses below are developed for covert networks in general.

H5. Narco terror and illicit drug networks have pre-existing social structures (kinship and peer).

H6. More endured covert networks with reputations are more security driven.

\section{Empirical model}

This study uses the SNA technique, a widely used method to identify relational patterns in social networks (Wasserman and Faust, 1994; Freeman et al. (1979); Hanneman and Riddle, 2005) to identify structural characteristics of sample networks in their prioritization between security and efficiency. Since a network is a system of interconnected members/actors/nodes (Zech and Gabbay, 2016: 216), SNA is a particularly effective analysis tool for exploring structural pattern of covert networks (Sparrow, 1991; McGloin and Kirk, 2010; Van der Hulst, 2009). Use of SNA in countering dark networks, largely stimulated by Sparrow's (1991) seminal work, has led academia to dwell on elucidating structural inferences on criminal (Natarajan, 2000, 2006; Baker and Faulkner, 1993; Bright et al., 2012) and terrorist groups (Koschade, 2006; Enders and Su, 2007; Krebs, 2002; Mayntz, 2004) or both (Belli et al., 2015; Crossley et al., 2012; Morselli et al., 2007; Milward and Raab, 2006; Rodriguez, 2009).

This study utilizes SNA metrics below-that were used by empirical studies cited in this research-in identifying topographies and structural characteristics of sample networks to analyze, in general, their behavior towards the security-efficiency tradeoff and to identify, in particular, whether or not DGNs and NTNs approach the security-efficiency trade-off differently.

It should be noted that network metrics, such as density, average nodal degree, clustering coefficient and etc., will vary greatly in networks of small size in adding/deleting a link/node. Density scores, for instance, tend to decrease as the size of a network increases (Everton, 2008: 98; Morselli et al., 2007: 148). So, when network densities are analyzed, their network size are taken into consideration and networks with similar size are compared. In this regard, this study also resorts to other metrics to supplement and complement the analyses of structural characteristics and topographies of sample networks.

Density reflects the degree of connectedness (network cohesion) among members of a network. Denser networks mean more incidence among members and in dense networks it is easier for people to monitor the behavior of others (policing each other) and prevent defection. Formally density measures the cohesion/connectedness of a network through the total number of observed ties within a network divided by the maximum possible number of ties ranging from 0.0 to 1.0 Freeman et al. (1979).

Average nodal degree is a supplementary metric to density estimating social connectedness by calculating the average number of lines/ neighbors each node/actor has (Wasserman and Faust, 1994: 101-3). A higher average nodal degree indicates a more intense relationship (Knoke and Young, 2008: 59).

Average Geodesic Distance is the average length of the shortest path ("optimal" connection) between two actors (Everton, 2008: 200) indicating how close actors are (Hanneman and Riddle, 2005: 81). The shorter the distance the more connected the actors are in the network.

Clustering Coefficient measures the average densities of neighborhoods of all actors in a network (Hanneman and Riddle, 2005), identifying the existence of densely knit clusters, known as the small world phenomena (Watts, 1999).

Transitivity measures the completed triads, two nodes in a network that are connected by an edge (Hanneman and Riddle, 2005: 93). It denotes connectedness of edges in a network structure.

Degree centrality is a count of the number of an actor's adjacent direct relational tie(s) (Everton, 2008:15; Wasserman and Faust, 1994; Freeman et al. (1979) indicating that the more direct ties an actor has the more central and powerful that actor is in the network. Likewise, the overall degree centrality for the network indicates the average score of all members' degrees in the network.

Closeness centrality indicate how close/far each actor is to all other actors in a network according to the average path distance Freeman et al. (1979). For actor-by-actor scores, a higher index indicates to greater distance of an actor from others. It measures the ability of a network member/node to reach other members/nodes in the minimum number of steps (Jordan et al., 2008). So, average closeness (normalized percentage) indicates how actors are close to each other.

Betweenness centrality estimates the extent to which each actor lies on the shortest path between all other actors in a network. This metric measures the intermediate (brokerage) positions of actors for coordinating and controlling relationships in the network and a higher index indicates greater intermediation (Wasserman and Faust, 1994; Everton, 2008:15; Freeman et al. (1979). It simply denotes the frequency of one node appearing between two other nodes (Jordan et al., 2008). Betweenness centrality keeps an actor active but from a more discreet position in the network (Morselli, 2010: 390).

Constraint (Structural Holes) is the metric measuring the involvement of actors in incomplete triads. Low constraint means more structural holes offering brokering options/positions to the actor and vice versa (Everton, 2008: 154). It is mostly used to identify the significant relational ties to disrupt a covert network (disruption points).

Multiplicative Coreness (continuous) uses a $0-1$ index to indicate how an actor is close to or correlated with a core preference pattern. An index score closer to 1 means increased correlation with core pattern. Coreness indicates a single individual's (node) position vis-à-vis a core in the network.

Core-peripheriness (correlation) at the network level, or overall coreness, refers to a measure of the correlation between tie strength and joint centrality. The index is higher if central actors tend to have strong ties with each other while peripheral actors tend to have weak ties (for all pairs of nodes) with one another (Borgatti and Everett, 2000). In the end, this metric identifies how network members are structure and attached to existing core(s) in a network.

Along the same lines, network level group centralization measures are plotted to analyze the generic characteristics and make up of networks. These include, for instance, the group level degree centralization that examines how centralized/hierarchical or decentralized/flat a network structure is on a $0-100$ percentage scale (normalized scores are used for comparison purpose), in which more variation (meaning few actors with high centrality scores) yields higher network centralization scores (Everton, 2008: 100; Wasserman and Faust, 1994: 176). Centralization is a complementary metric to density and degree centrality. While density describes the overall cohesion, centralization denotes how such cohesion is organized around specific actor/node as focal points.

In addition, sociograms (graphic representation of social links in a visual network structure) are plotted to supplement the general understanding about these networks. The size of nodes (actors) and labels in sociograms are developed based on networks' betweenness attributes (as a centrality and power measure) to identify pivotal players and nodes that have kinship structure are denoted with yellow color. All analyses have been conducted and results are plotted using UCINET and NetDraw software. 
Results

This section, first, briefly introduces each sample network, NT and DG cases, and discusses certain actor-by-actor and network level SNA metrics-related to the developed hypotheses-such as cohesion and connectedness, centrality, core-peripheriness. It also identifies type of trafficked drug, kinship structures, positions of key players and for NTNs positions of terrorist members to discuss each network's prioritization between security and efficiency. Yet, it provides arrests rates, and structural positioning of arrested and fugitive members of these dark networks ("Y" denotes arrested and "N" denotes fugitives in individual tables).

Next, it compares and contrasts the structural characteristics of DGNs and NTNs to identify whether or not a difference exists in prioritizing between security and efficiency. ${ }^{7}$ Overall results in tested hypotheses and related prior research are thematically discussed in the following section.

\section{Narco-terror networks}

NT\#01: This case refers to a 21-member network, 10 of whom are Iranian, trafficking morphine base from Iran to Europe through Turkey. ${ }^{8}$ Only two members are linked to the PKK. As plotted in the sociogram (Fig. 1), NT\#01 has a polycentric structure with two separate dense subgroups that are tied to one another through the key players. In each sub-group, core and clique-like dense relations exist with shorter distance, evident in a relatively higher clustering coefficient (0.64) and transitivity (22.61) as depicted in Table 12, and strong ties (bolder lines) as indicated in Fig. 1.

There exist four key players, one of whom is the leader, T6-ORL, linked to the PKK. T6-ORL plays a critical brokerage role between the two main clusters as evident in the highest (actor level) betweenness (56.633), lowest farness (37), and one of the lowest constraint (0.379) as plotted in Table 1. This indicates the importance of his position for the efficiency and survival of the network, in which his removal would structurally destabilize the bulk of the network. Another key player, 11HLM, has the highest coreness value being in the pivotal position. So key players either have critical brokerage (betweenness) or pivotal (degree centrality) positions to effectively control and coordinate the network, constituting structurally weak points.

Overall density is 0.20 with two weakly tied separate clusters indicating very high average betweenness centrality (77.19 percent) as depicted in Table 11. A high average nodal degree of 4.09 and a relatively short average geodesic distance of 2.47 indicate high connectedness/transitivity in each of the sub-groups. In line with this, strong kinship structures are denoted with yellow nodes in Fig. 1, in which, for instance, actors 19 and 18 are brothers, and the leader, T6ORL, is the son of actor 14. In light of these, the NT\#01 is more efficiency driven than security. Except two Iranians, T20 (kinship tie with actor 5) and 21 (kinship tie with T6-ORL and 14) that are positioned in the peripheries of each core (see sociogram in Fig. 1), all network members were captured and arrested.

NT\#02: This case refers to an all Turkish, 17-member network, two of whom are terrorists (T5-HLM and T3-ORL), trafficking heroine and morphine base. Table 2 plots actor-by-actor scores and as seen in the table, both terrorists have strategic level positions and reflect the highest power by their degree ( 6 and 5 respectively) and betweenness

\footnotetext{
${ }^{7}$ To make the analyses easy-to-follow, table sequence is adjusted to the case numbers (e.g., actor-by-actor results for \#01 are plotted in Table 1 and the sociogram is plotted in Fig. 1). The general results for centrality, cohesion and connectedness, and pre-existing ties are plotted in Tables 11,12, and 13 respectively.

${ }^{8}$ The PKK not only has a branch in Iran, but its workforce also contains Iranian, Iraqi, and Syrian nationalities (see Ünal, 2012).
}

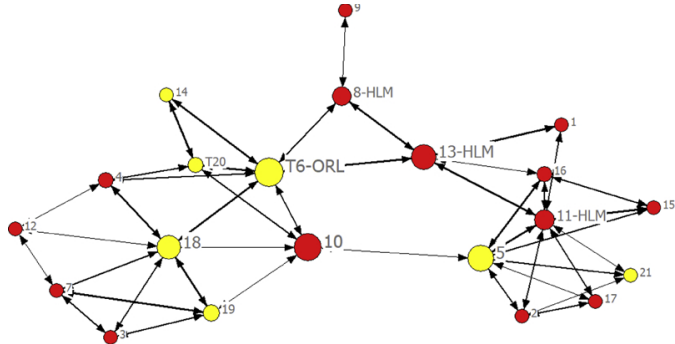

Fig. 1. Sociogram for the Network NT\#01.

Table 1

Network NT\#01 - Actor-by-actor power and centrality scores.

\begin{tabular}{|c|c|c|c|c|c|c|}
\hline Node & Degree & Between & Close & Constraint & Coreness & Arrest \\
\hline 1 & 2 & 0 & 52 & 1.125 & 0.141 & $\mathrm{Y}$ \\
\hline 2 & 4 & 0.333 & 53 & 0.74 & 0.296 & $\mathrm{Y}$ \\
\hline 3 & 3 & 0 & 58 & 0.926 & 0.031 & $\mathrm{Y}$ \\
\hline 4 & 4 & 5.817 & 49 & 0.642 & 0.053 & $\mathrm{Y}$ \\
\hline 5 & 7 & 48.683 & 41 & 0.399 & 0.459 & $\mathrm{Y}$ \\
\hline T6-ORL & 7 & 56.633 & 37 & 0.379 & 0.119 & $\mathrm{Y}$ \\
\hline 7 & 4 & 1 & 57 & 0.704 & 0.036 & $\mathrm{Y}$ \\
\hline 8-HLM & 3 & 19 & 46 & 0.611 & 0.063 & $\mathrm{Y}$ \\
\hline 9 & 1 & 0 & 65 & 1 & 0.011 & $\mathrm{Y}$ \\
\hline 10 & 5 & 53.75 & 37 & 0.451 & 0.142 & $\mathrm{Y}$ \\
\hline 11-HLM & 8 & 25.367 & 44 & 0.372 & 0.553 & $\mathrm{Y}$ \\
\hline 12 & 3 & 0.7 & 57 & 0.84 & 0.031 & $\mathrm{Y}$ \\
\hline 13-HLM & 5 & 40.483 & 39 & 0.482 & 0.219 & $\mathrm{Y}$ \\
\hline 14 & 2 & 0 & 55 & 1.125 & 0.033 & $\mathrm{Y}$ \\
\hline 15 & 3 & 0 & 54 & 0.926 & 0.248 & $\mathrm{Y}$ \\
\hline 16 & 4 & 3.45 & 48 & 0.704 & 0.288 & $\mathrm{Y}$ \\
\hline 17 & 3 & 0 & 54 & 0.926 & 0.249 & $\mathrm{Y}$ \\
\hline 18 & 7 & 39.967 & 41 & 0.412 & 0.084 & $\mathrm{Y}$ \\
\hline 19 & 4 & 7.783 & 49 & 0.704 & 0.053 & $\mathrm{Y}$ \\
\hline T20 & 4 & 5.033 & 46 & 0.684 & 0.063 & $\mathrm{~N}$ \\
\hline 21 & 3 & 0 & 54 & 0.926 & 0.249 & $\mathrm{~N}$ \\
\hline
\end{tabular}

Table 2

Network NT\#02 - Actor-by-actor power and centrality scores.

\begin{tabular}{lllllll}
\hline Node & Degree & Between & Close & Constraint & Coreness & Arrest \\
\hline 1 & 1 & 0 & 63 & 1 & 0.005 & $\mathrm{Y}$ \\
2 & 1 & 0 & 63 & 1 & 0.005 & $\mathrm{Y}$ \\
T3-ORL & 6 & 82 & 29 & 0.223 & 0.905 & $\mathrm{Y}$ \\
4 & 3 & 29 & 48 & 0.333 & 0.032 & $\mathrm{Y}$ \\
T5-HLM & 5 & 46 & 36 & 0.2 & 0.175 & $\mathrm{Y}$ \\
6 & 4 & 45 & 36 & 0.25 & 0.169 & $\mathrm{Y}$ \\
7 & 1 & 0 & 51 & 1 & 0.028 & $\mathrm{Y}$ \\
8 & 1 & 0 & 51 & 1 & 0.027 & $\mathrm{Y}$ \\
9 & 2 & 8 & 43 & 0.5 & 0.056 & $\mathrm{Y}$ \\
10 & 2 & 15 & 49 & 0.5 & 0.028 & $\mathrm{Y}$ \\
11 & 2 & 0 & 40 & 0.785 & 0.183 & $\mathrm{Y}$ \\
12 & 1 & 0 & 44 & 1 & 0.15 & $\mathrm{Y}$ \\
13 & 1 & 0 & 64 & 1 & 0.005 & $\mathrm{Y}$ \\
14 & 3 & 39 & 37 & 0.512 & 0.188 & $\mathrm{Y}$ \\
15 & 1 & 0 & 44 & 1 & 0.15 & $\mathrm{Y}$ \\
16 & 1 & 0 & 51 & 1 & 0.028 & $\mathrm{Y}$ \\
17 & 1 & 0 & 51 & 1 & 0.028 & $\mathrm{Y}$ \\
\hline
\end{tabular}

(82 and 46) indicating that they have the highest number of direct ties to others and lie on the shortest path between all other actors. These actors reflect central hubs with high brokerage roles to control and coordinate the flow of information and resources (constraint scores of 0.2 and 0.22 respectively). As shown in Fig. 2 (yellow nodes) and Table 13, NT\#02 has strong trust ties. The star-like structure indicates that terrorists constitute hubs tied to one another with offenders directly connected only to them showing strict control and authority. For a network size of 17, the average geodesic distance of 2.94 can be deemed higher than other sample networks, indicating increased 


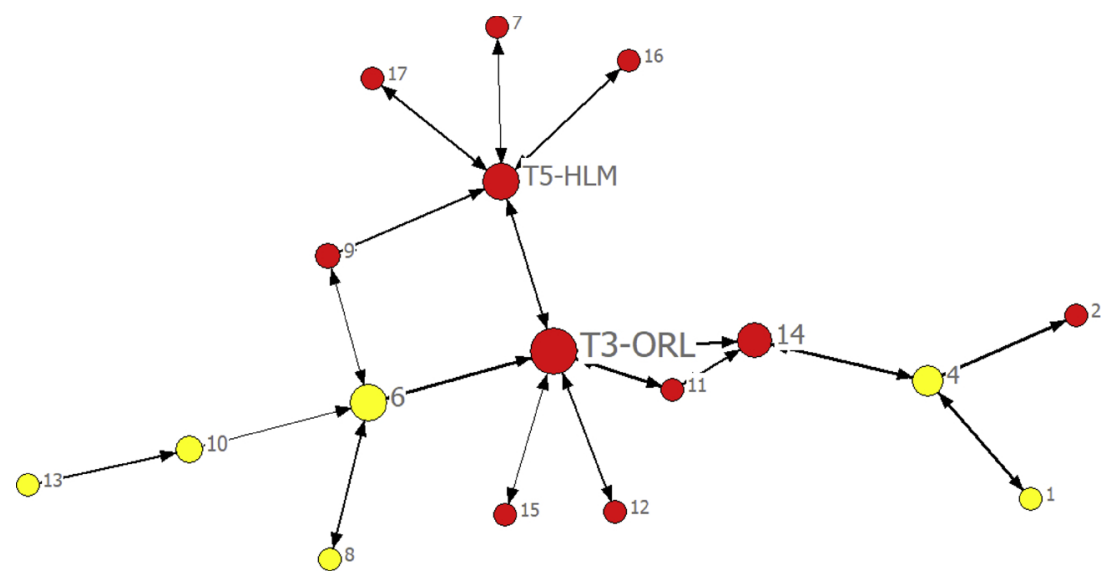

Fig. 2. Sociogram for the Network NT\#02.

Table 3

Network NT\#03 - Actor-by-actor power and centrality scores.

\begin{tabular}{lllllll}
\hline Node & Degree & Between & Close & Constraint & Coreness & Arrest \\
\hline 1 & 3 & 0 & 24 & 0.926 & 0.236 & $\mathrm{Y}$ \\
2 & 1 & 0 & 34 & 1 & 0.042 & $\mathrm{Y}$ \\
3 & 2 & 0 & 26 & 1.125 & 0.179 & $\mathrm{Y}$ \\
4 & 1 & 0 & 33 & 1 & 0.05 & $\mathrm{Y}$ \\
5 & 2 & 0 & 26 & 1.125 & 0.114 & $\mathrm{Y}$ \\
6 -HLM & 5 & 12.667 & 21 & 0.514 & 0.288 & $\mathrm{Y}$ \\
7 & 8 & 21.417 & 18 & 0.361 & 0.43 & $\mathrm{Y}$ \\
8 & 5 & 4.5 & 23 & 0.513 & 0.222 & $\mathrm{Y}$ \\
9 & 2 & 0 & 26 & 1.125 & 0.179 & $\mathrm{Y}$ \\
$10-\mathrm{DP}$ & 9 & 28.667 & 17 & 0.333 & 0.576 & $\mathrm{~N}$ \\
T11-DP & 4 & 0.25 & 24 & 0.74 & 0.227 & $\mathrm{~N}$ \\
T12-DP & 4 & 0.25 & 24 & 0.74 & 0.227 & $\mathrm{~N}$ \\
13 & 4 & 0.25 & 24 & 0.74 & 0.227 & $\mathrm{~N}$ \\
14 & 4 & 12 & 22 & 0.583 & 0.244 & $\mathrm{~N}$ \\
\hline
\end{tabular}

distance and therefore assuring greater security evident in the lowest scores of density (0.13) and clustering coefficient (0.17).

In the end, terrorists constitute the core with high centrality and power, however, yielding structural holes with high level brokerage opportunities as evident in higher network (degree) centralization (58.85 percent) around terrorist members (Table 11). While this implies reliance on efficiency through central hub control and coordination, low connectedness and cohesion reflects a security driven structure. For the NT\#02, security exists through lower direct ties among members which is considered optimal for a dark network in its initial stage in hostile environments (Lindelauf et al., 2009). However, all members were arrested.

NT\#03: This case refers to an all Turkish, 14-member network, two of whom are terrorists, trafficking heroine. As opposed to other NTNs, these terrorist members are positioned on the periphery acting as DPs. The organizational leader is not identifiable by the available data. There is a high-level manager (6-HLM) positioned on the periphery. There is another DP, 10-DP, has a central position (highest degree 9, betweenness 28.667 , coreness 0.576 , lowest distance 17 , and lowest constraint 0.33 ) as depicted in Table 3 . The overall network indicates a higher clustering coefficient (0.71), short paths (average geodesic distance of 1.88) and high connectedness with a 3.85 average nodal degree (Table 12). The sociogram (Fig. 3) indicates that the network NT\#03 has a core-periphery structure (core-peripheriness of 0.56) around the 10-DP with complete triads (transitivity of 21.25 percent) as reflected in Table 12.

Terrorist members (T12-DP and T11-DP) reflect a clique with strong ties (bolder lines in sociogram). This is expected given that T11-DP is the brother of T12-DP and the uncle of node 13 with most members (11 out of 14) having peer and friendship s as depicted in Table 13. The overall network structure, despite terrorists are positioned in the periphery and unidentified leaders, indicates more reliance on efficiency given the short paths and clusters attached to the network's core. In the end, out of 14, nine were arrested and the key player at the center (10DP) and PKK affiliated terrorists (T11-DP, T12-DP, 13, and 14) positioned on the periphery (five) were fugitives.

NT\#04: This case represents an all Turkish network trafficking hashish and heroine. It has more terrorist members than drug offenders. Out of 12 members, seven were linked to the PKK. No leader is identified, however two terrorists (T2 and T8) connect two subgroups (Fig. 4). The 12-HLM, as the only key player, is positioned on the periphery. Kinship ties exist among four members, two of whom are terrorists (yellow nodes). T2 has the pivotal role with more ties and shares high betweenness scores with T8 (34 and 26 respectively). Together they act as a gateway between two clusters and thus constitute structural holes (constraint scores of 0.4570 .469 ) as depicted in Table 4. The sub-group around "T2" indicates a clique (T2, 6, 11, 10).

With a moderate core-peripheriness $(0.56)$, the network NT\#03 has two clusters weakly tied with a relatively higher clustering coefficient (0.63) and transitive structure (22.64 percent) with the average path of 2.1 (Fig. 4 and Table 12). Almost all members had friendship ties before the network was formed, and four of them had kinship ties. The level of clustering and path length indicate reliance on efficiency but key players' positions, and high kinship and peer structure reflect striving for security. Except four, all other members were captured and arrested. In that, one of the key players (12-HLM) and one of the terrorists (T2) having blood ties with actors 6 and 7 that are all positioned on the periphery were fugitives.

NT\#05: This case represents an all Turkish, 10-member network, five of whom are terrorists, trafficking hashish and marijuana. As in other cases, the terrorist members are predominantly controlling the flow of information and resources. As shown in Table 5, T3-HLM indicates the most direct ties (7) and the shortest paths to all others (16.66). He also has strong ties with all other terrorists (T4, T5, T6, and T10). Terrorist members reflect strong kinship structures; T6, T4 and 7 are brothers.

As depicted in the sociogram (Fig. 5), the leader, 9-ORL, is less prominent in terms of degree centrality and positioned on the periphery with only two direct ties to two terrorist members, T3-HLM and T-6. The network has higher core-peripheriness (0.66) around T3-HLM (constraint of 0.397) as well as higher connectedness with transitivity of 25.64 percent, geodesic distance of 1.76 , and density of 0.38 (Table 12) which is in line with mid-level degree and closeness centralization (Table 11). Given relatively higher centralization, connectedness and short paths with relatively higher density, network NT\#05 seems to rely more on efficiency than security. All members, 


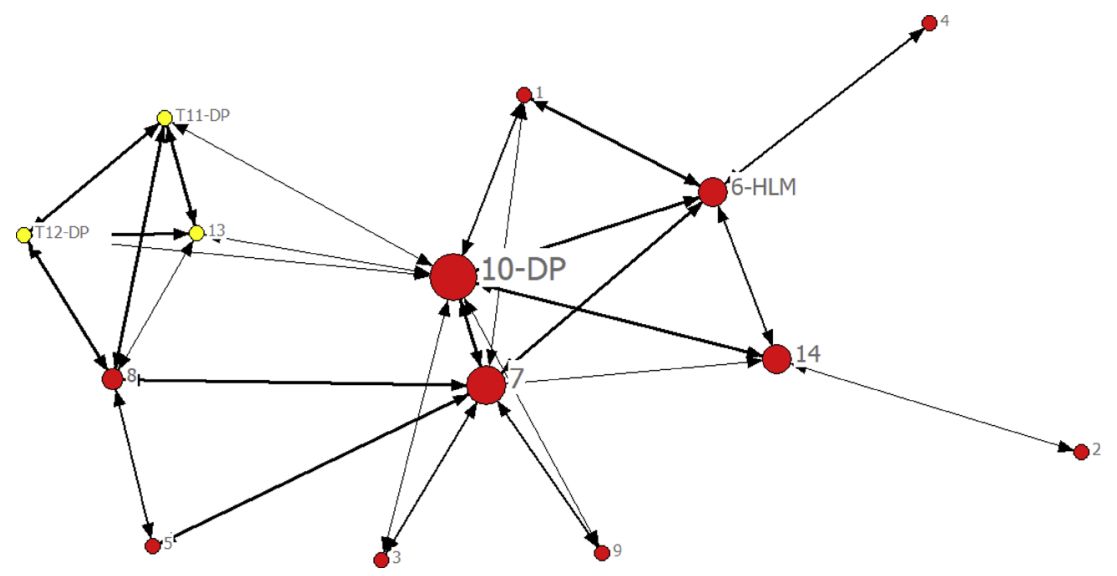

Fig. 3. Sociogram for the Network NT\#03.

except T5 (despite his position close to the core with direct ties and brokerage role), were captured and arrested including the leader positioned on the periphery.

\section{Illicit drug networks}

DG\#01: This case represents an all Turkish, 23-offender network trafficking heroine, hashish, and marijuana. Two of the members have kinship ties while 11 offenders had (bilateral and/or multilateral) peer and/or friend ties. As plotted in Fig. 6, two clear clusters exist controlled and bridged by the interconnected pivotal actors 22-ORL and 11-ORL as evident in their highest scores of coreness: 0.763 and 0.276 and lowest scores of constraint: 0.12 and 0.22 respectively (Table 6). As indicated with yellow nodes, there exist kinship relations between offenders 16 and 1 (brothers) and 11-ORL and actor 23.

As depicted in Table 12, the network DG\#01 is sparse with a lowdensity score of 0.14 compared to other sample networks and criminal networks of similar size in the literature (e.g., see Baker and Faulkner, 1993). Verifying the low connectedness, the average nodal degree (3), degree centrality (14.62 percent), transitivity (6.74 percent) all indicate less ties among members. The star-like structure shows a lower clustering coefficient of 0.407 (to compare also see Krebs, 2002) and a high betweenness centrality score of 65.97 percent (Table 11) as also seen in the network's sociogram (Fig. 6). In the end, as argued by Lindelauf et al. (2009), central hubs controlled by the 22-ORL and 11-ORL keep the path lengths short to minimize chance of interception, particularly in the initial stage of operation in a hostile environment. However, more ties to a central actor cause vulnerability (see Enders and Su, 2007) to detection due to increased incidence of action for one node.
Table 4

Network NT\#04 - Actor-by-actor power and centrality scores.

\begin{tabular}{lllllll}
\hline Node & Degree & Between & Close & Constraint & Coreness & Arrest \\
\hline T1 & 2 & 0 & 29 & 1.125 & 0.052 & Y \\
T2 & 6 & 34 & 16 & 0.457 & 0.582 & Y \\
T3 & 1 & 0 & 33 & 1 & 0.052 & Y \\
T4 & 3 & 10 & 23 & 0.611 & 0.226 & Y \\
T5 & 1 & 0 & 30 & 1 & 0.04 & Y \\
6 & 3 & 0 & 23 & 0.882 & 0.37 & N \\
7 & 1 & 0 & 31 & 1 & 0.119 & N \\
T8 & 4 & 26 & 20 & 0.469 & 0.172 & Y \\
T9 & 2 & 0 & 24 & 1.125 & 0.271 & N \\
10 & 4 & 3 & 21 & 0.732 & 0.297 & Y \\
11 & 5 & 11 & 21 & 0.492 & 0.509 & Y \\
$12-H L M$ & 2 & 0 & 29 & 1.125 & 0.052 & N \\
\hline
\end{tabular}

Table 5

Network NT\#05 - Actor-by-actor power and centrality scores.

\begin{tabular}{lllllll}
\hline Node & Degree & Between & Close & Constraint & Coreness & Arrest \\
\hline 1 & 2 & 0 & 18 & 1.125 & 0.13 & Y \\
2 & 2 & 0 & 18 & 1.125 & 0.13 & Y \\
T3-HLM & 7 & 16.667 & 11 & 0.397 & 0.537 & Y \\
T4 & 5 & 10 & 13 & 0.491 & 0.363 & Y \\
T5 & 4 & 0.667 & 14 & 0.74 & 0.377 & N \\
T6 & 6 & 6.667 & 12 & 0.487 & 0.492 & Y \\
7 & 2 & 0 & 18 & 1.125 & 0.169 & Y \\
8 & 1 & 0 & 21 & 1 & 0.07 & Y \\
$9-$-ORL & 2 & 0 & 17 & 1.125 & 0.206 & Y \\
T10 & 3 & 0 & 16 & 0.926 & 0.295 & Y \\
\hline
\end{tabular}

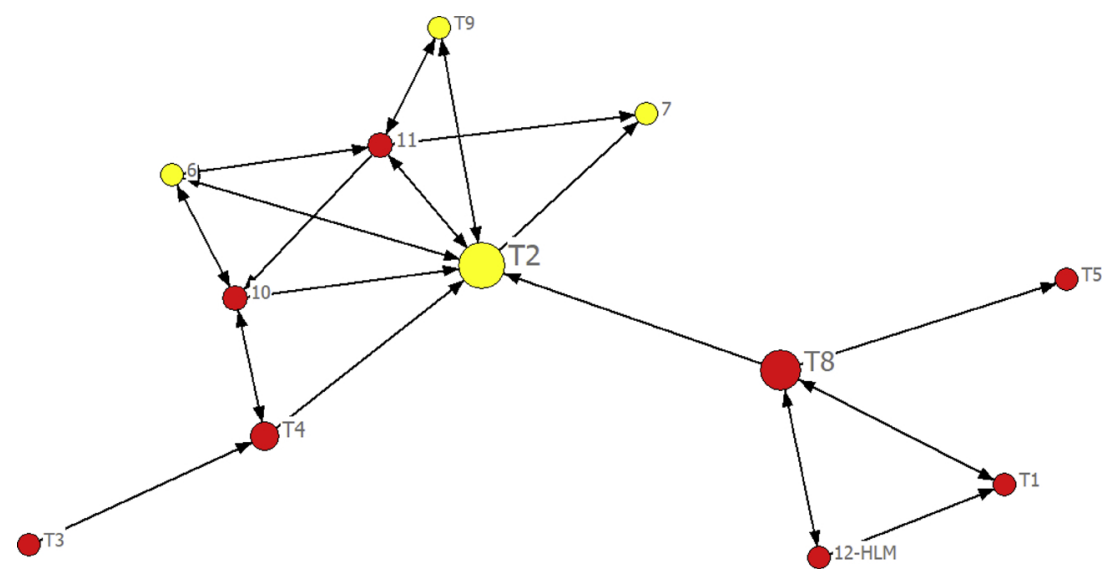

Fig. 4. Sociogram for the Network NT\#04. 


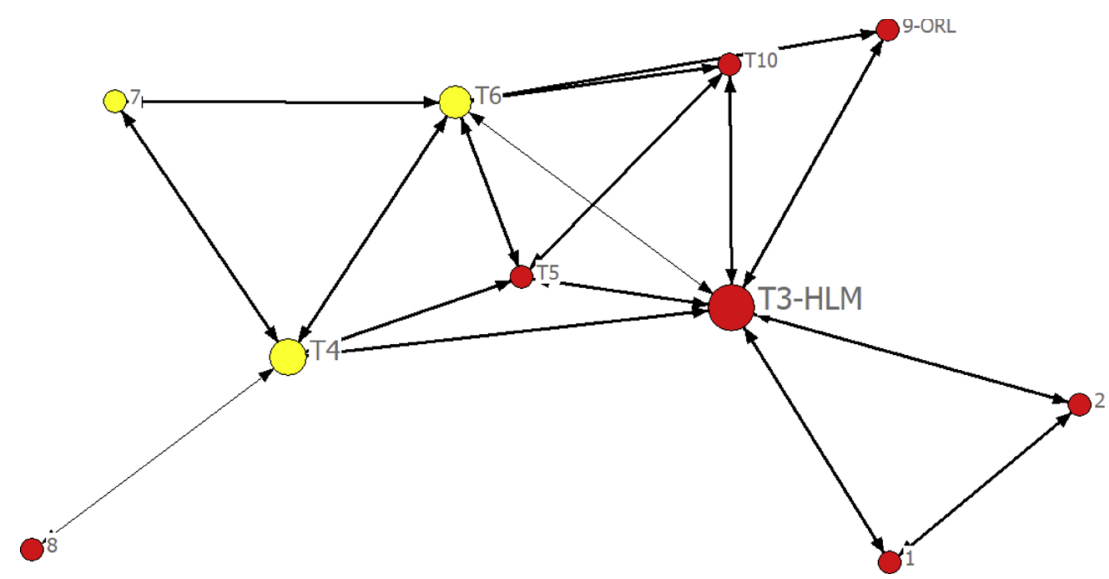

Fig. 5. Sociogram for the Network NT\#05.

The highest actor scores of betweenness (128.5 and 104.83 respectively) and the lowest constraint ( 0.12 and 0.22$)$ would suggest elimination of the entire network when compromised. Despite security oriented structure, such as low density, out of 23, 19 members including key players were captured and arrested. In that, the actors 8, 9, 20, and 18-DP were fugitives who were tied to the core that yields a star-like structure in which they have only one direct tie to the central hub (22ORL) and the actor 9 was already an isolate (relational tie is not identified based on available data).

DG\# 02: This case represents a 12-offender network trafficking the synthetic drug captagon. The network is all Turkish except one Iranian. Two of the members are relatives and almost of all are friends and peers. As plotted in Fig. 7, the network has a core that is controlled and coordinated by one key player, 1-HLM, who, with many strong ties acts as a focal point with the highest brokerage and power scores (e.g., degree: 9, betweenness: 27.23, coreness: 0.571 ) as depicted in Table 7 . The leader, 11-ORL, also has a central position with many direct ties. Two key players are directly connected and close to each other exerting power and authority to control of the flow of resources and information. However, connected edges around the actors tied to 11-ORL decrease the strictness of control to some degree as evident in transitivity of 27.69 percent, high closeness centralization (63.49 percent), and low average betweenness centrality (8.33 percent only), as shown in Tables 11 and 12.

As shown in Table 12, the network DG\#02 also has a higher clustering coefficient of 0.708 indicating denser clusters with more ties in shorter distance (average of 1.833) when compared to, for instance, 19 hijackers analyzed by Krebs (2002): clustering coefficient of 0.41 and
Table 6

Network DG\#01 - Actor-by-actor power and centrality scores.

\begin{tabular}{|c|c|c|c|c|c|c|}
\hline Node & Degree & Between & Close & Constraint & Coreness & Arrest \\
\hline 1 & 1 & 0 & 94 & 1 & 0.008 & $\mathrm{Y}$ \\
\hline 2 & 6 & 30.667 & 67 & 0.407 & 0.231 & $\mathrm{Y}$ \\
\hline 3 & 3 & 6 & 70 & 0.521 & 0.188 & $\mathrm{Y}$ \\
\hline 4 & 3 & 3.167 & 77 & 1.042 & 0.069 & $\mathrm{Y}$ \\
\hline 5 & 2 & 4 & 72 & 0.5 & 0.079 & $\mathrm{Y}$ \\
\hline 6 & 1 & 0 & 76 & 1 & 0.131 & $\mathrm{Y}$ \\
\hline 7 & 3 & 1.833 & 71 & 0.882 & 0.16 & $\mathrm{Y}$ \\
\hline 8 & 1 & 0 & 76 & 1 & 0.098 & $\mathrm{~N}$ \\
\hline 9 & 0 & 0 & 506 & 0 & 0.011 & $\mathrm{~N}$ \\
\hline 10 & 2 & 0 & 75 & 1.125 & 0.162 & $\mathrm{Y}$ \\
\hline 11-ORL & 10 & 104.833 & 56 & 0.22 & 0.763 & $\mathrm{Y}$ \\
\hline 12 & 3 & 18.333 & 68 & 0.604 & 0.107 & $\mathrm{Y}$ \\
\hline 13 & 2 & 0 & 75 & 1.125 & 0.169 & $\mathrm{Y}$ \\
\hline 14 & 2 & 0 & 75 & 1.125 & 0.16 & $\mathrm{Y}$ \\
\hline 15 & 1 & 0 & 76 & 1 & 0.047 & $\mathrm{Y}$ \\
\hline 16 & 2 & 20 & 74 & 0.5 & 0.048 & $\mathrm{Y}$ \\
\hline 17 & 3 & 4.667 & 65 & 0.821 & 0.228 & $\mathrm{Y}$ \\
\hline 18-DP & 1 & 0 & 76 & 1 & 0.047 & $\mathrm{~N}$ \\
\hline 19 & 1 & 0 & 76 & 1 & 0.047 & $\mathrm{Y}$ \\
\hline 20 & 1 & 0 & 76 & 1 & 0.047 & $\mathrm{~N}$ \\
\hline 21 & 2 & 0 & 86 & 1.235 & 0.068 & $\mathrm{Y}$ \\
\hline 22-ORL & 10 & 128.5 & 56 & 0.12 & 0.276 & $\mathrm{Y}$ \\
\hline 23 & 2 & 0 & 75 & 0.889 & 0.173 & $\mathrm{Y}$ \\
\hline
\end{tabular}

average path length of 4.75 . This is also evident in the higher density (0.36) and higher average nodal degree (4) indicating the network's reliance on efficiency to ensure effective communication. Despite this,

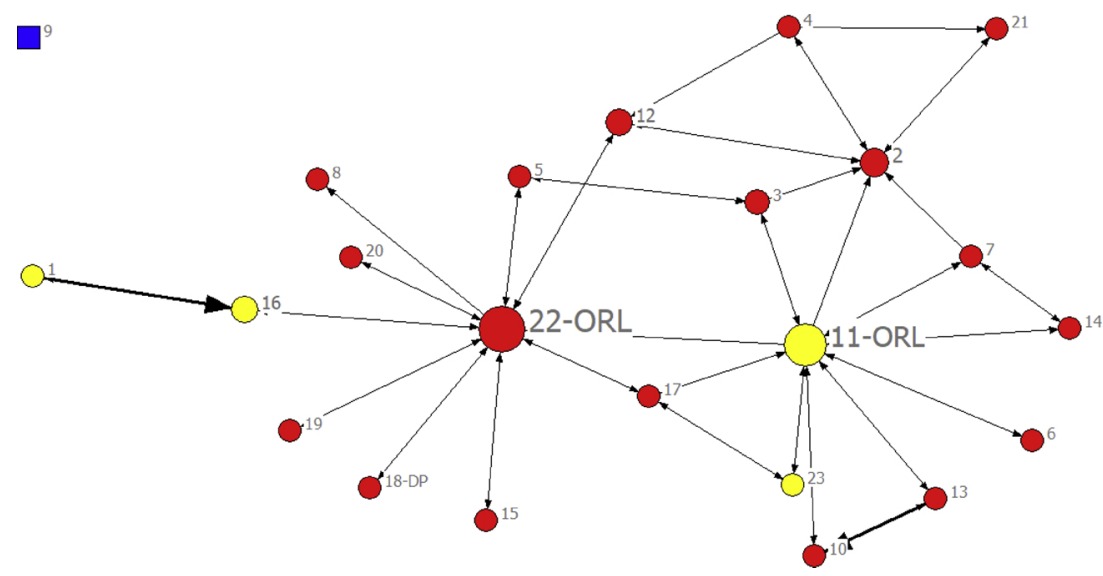

Fig. 6. Sociogram for the Network DG\#01. 


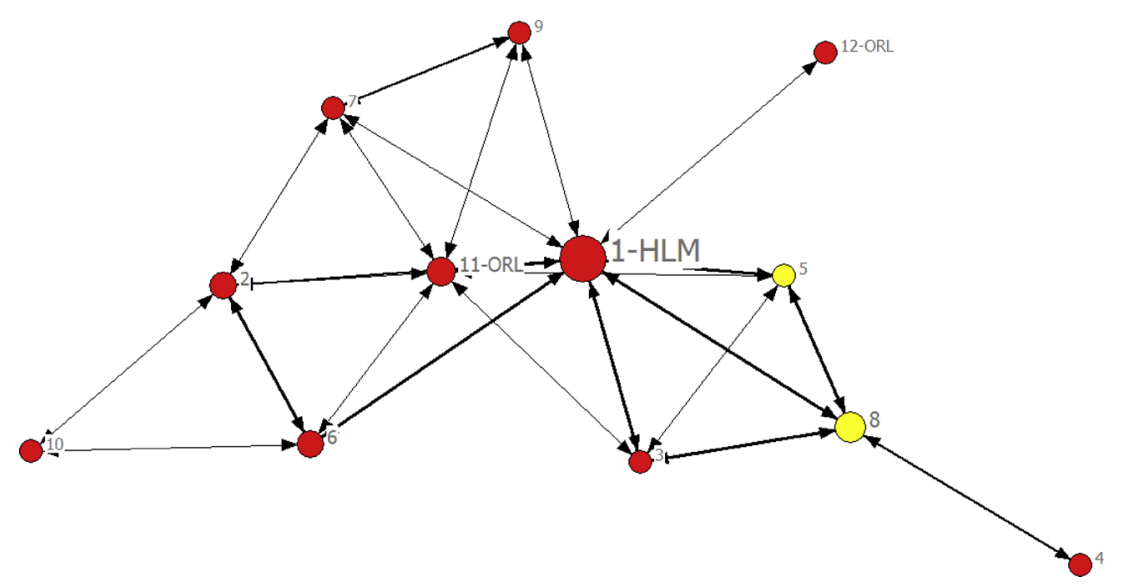

Fig. 7. Sociogram for the Network DG\#02.

Table 7

Network DG\#02 - Actor-by-actor power and centrality scores.

\begin{tabular}{lllllll}
\hline Node & Degree & Between. & Close. & Constraint & Coreness & Arrest \\
\hline 1 -HLM & 9 & 27.233 & 13 & 0.3 & 0.571 & $\mathrm{Y}$ \\
2 & 5 & 5.433 & 18 & 0.46 & 0.298 & $\mathrm{Y}$ \\
3 & 4 & 0.667 & 19 & 0.542 & 0.264 & $\mathrm{Y}$ \\
4 & 1 & 0 & 29 & 1 & 0.033 & $\mathrm{~N}$ \\
5 & 4 & 0.667 & 19 & 0.542 & 0.264 & $\mathrm{Y}$ \\
6 & 4 & 3.9 & 19 & 0.502 & 0.249 & $\mathrm{Y}$ \\
7 & 4 & 0.533 & 19 & 0.541 & 0.277 & $\mathrm{Y}$ \\
8 & 4 & 10 & 19 & 0.435 & 0.196 & $\mathrm{Y}$ \\
9 & 3 & 0 & 21 & 0.596 & 0.227 & $\mathrm{Y}$ \\
10 & 2 & 0 & 27 & 0.751 & 0.092 & $\mathrm{Y}$ \\
11 -ORL & 7 & 6.567 & 16 & 0.388 & 0.446 & $\mathrm{~N}$ \\
$12-$ ORL & 1 & 0 & 23 & 1 & 0.096 & $\mathrm{~N}$ \\
\hline
\end{tabular}

the organizational leader 12-ORL is less prominent having only one tie to the 1-HLM, which isolate him and maintain his secrecy/security. Only actors 8 and 5 have blood ties, but many intense relationships exist in this network (bolder lines). Out of 12 offenders, 9 of them were captured and arrested while both organizational leaders (11-ORL and 12-ORL) and actor 4 were fugitives. While 12-ORL and actor 4 were positioned on the periphery, 11-ORL was at the network's core with high degree centrality (direct ties) to many others including the key player 1-HLM as plotted in Fig. 7 (sociogram).

DG\# 03: This case represents an all Turkish network trafficking the synthetic drug ecstasy. The network has 11 offenders, half of whom have kinship ties and almost all of whom have friendship ties. As Fig. 8 plots, the 7-ORL has the central (coreness: 0.698) and most powerful position in the network, evident in actor-by-actor scores reflected in
Table 8

Network DG\#03 - Actor-by-actor power and centrality scores.

\begin{tabular}{lllllll}
\hline Node & Degree & Between. & Close. & Constraint & Coreness & Arrest \\
\hline 1 & 4 & 16 & 16 & 0.45 & 0.23 & Y \\
2 & 2 & 0 & 20 & 0.676 & 0.207 & Y \\
3 & 2 & 0 & 20 & 0.707 & 0.196 & Y \\
4 & 2 & 0 & 24 & 0.953 & 0.053 & Y \\
5 -HLM & 3 & 0 & 19 & 0.639 & 0.279 & Y \\
6 & 2 & 0 & 24 & 0.953 & 0.053 & Y \\
7-ORL & 8 & 26.5 & 12 & 0.322 & 0.698 & Y \\
8-HLM & 4 & 1 & 18 & 0.58 & 0.319 & Y \\
9 & 2 & 0 & 20 & 0.879 & 0.164 & Y \\
10 & 5 & 5.5 & 15 & 0.48 & 0.373 & Y \\
11 & 2 & 0 & 20 & 0.879 & 0.164 & $\mathrm{~N}$ \\
\hline
\end{tabular}

Table 8. Two of the high-level managers, 8-HLM and 5-HLM, are positioned at the periphery (lower coreness of 0.319 and 0.279 respectively). Despite that HLMs are strongly tied (bolder lines) to the organizational leader.

The network seems to be relying more on efficiency given the network's high clustering coefficient of 0.795 , transitivity percentage of 21.95 , average nodal degree of 3.273 , and shorter average geodesic distance of 1.891 (Table 12). The connectedness among these actors is not surprising given that 5-HLM is brother of 8-HLM, 7-ORL is uncle of 5-HLM, and 8-HLM has a blood tie (through marriage) to 10 .

Overall, DG\#03's density (0.33) is relatively high for a dark network size of 11 (to compare, see Natarajan, 2006; Baker and Faulkner, 1993). Offenders are relatively closer (closeness centrality 54.91 percent) to each other on average. As reflected in Table 11, group

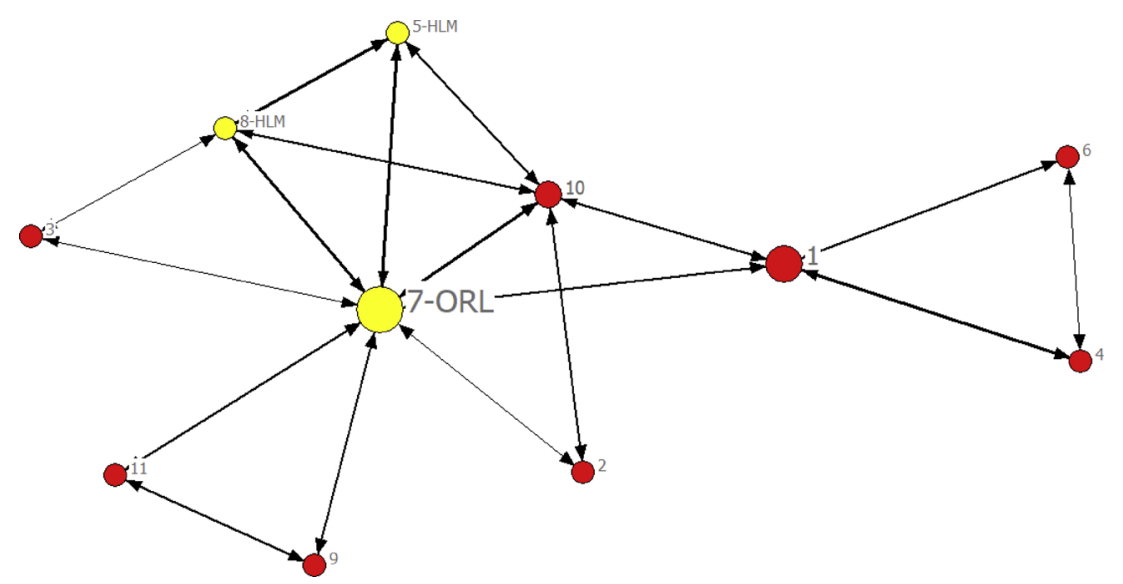

Fig. 8. Sociogram for the Network DG\#03. 


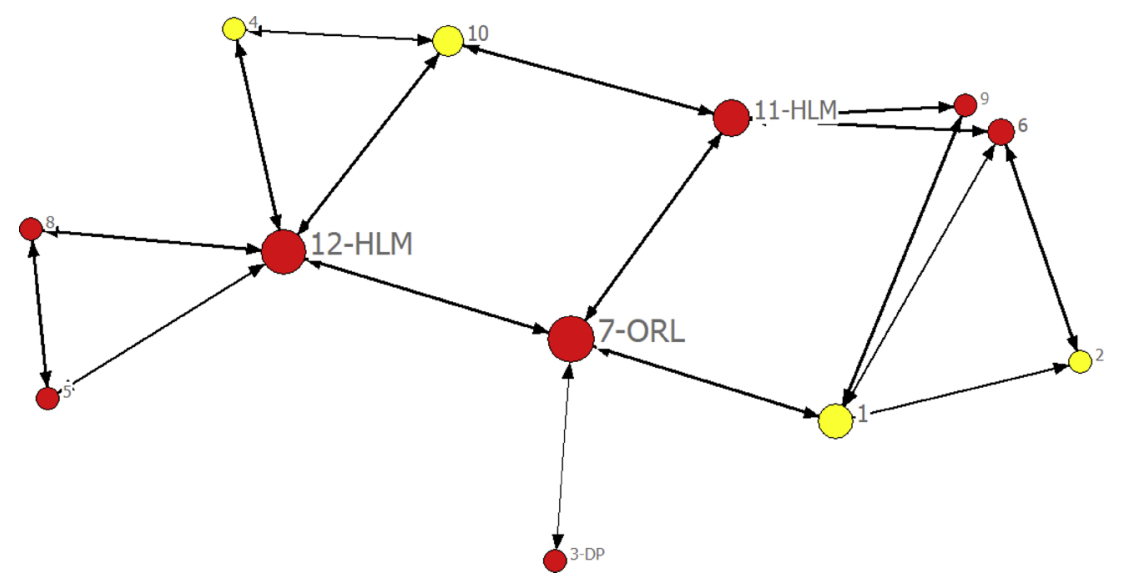

Fig. 9. Sociogram for the Network DG\#04.

centralization scores, e.g., degree centralization of 57.78 percent and closeness centralization of 66 percent, indicate a centralized structure indicating a more efficiency oriented structure. Hence, except actor 11 that is positioned on the periphery, all offenders were captured.

DG\#04: This case represents an all Turkish network trafficking heroine. The network is comprised of 12 offenders with one leader and two high level managers. Four members have kinship ties. Most individuals are friends and peers (11 out of 12). The overall network sociogram (Fig. 9) indicates a snake-like structure with no clearly identifiable central core. This is clearly reflected in the network's low levels of group centralization figures, such as centralization of degree (23.64 percent), closeness (35.09 percent), and betweenness (33.72 percent). In line with this, the network sociogram does not picture a dense cluster, evident in the low clustering coefficient of 0.458 .

Yet, given the small size of 12 , the network has a relatively higher average geodesic path distance of 2.241 (being relatively more security driven), when compared to networks DG\#02 (1.833) and NT\#04 (2.1) of the same size. Similar to DG\#02 and NT\#04, this network is relatively sparse with a density of 0.26 , betweenness centrality of 12.42 percent and the lowest core-peripheriness of 0.341 (Tables 11 and 12). Overall, network DG\#04, from an organizational security perspective, reflects lower connectedness that tends to reflect a horizontal/flattened structure compared to other sample networks indicating a relatively more security oriented structure than efficiency. However, except leader 7-ORL positioned at the network's core, all other network members were captured and arrested as depicted in Table 9.

DG\#05: The final case represents a 13-offender network trafficking heroine. The network is comprised of 5 Turks, 3 Bulgarians, 3 Syrians, with no kinship ties. The overall structure, as plotted in sociogram (Fig. 10), indicates a core centered around high-level manager 10-HLM. At the same time, actors on the periphery are connected to each other in addition to their direct ties to 10 -HLM. This is evident by the relatively

Table 9

Network DG\#04 Actor-by-actor power and centrality scores.

\begin{tabular}{lllllll}
\hline Node & Degree & Between & Close & Constraint & Coreness & Arrest \\
\hline 1 & 4 & 11 & 22 & 0.377 & 0.267 & $\mathrm{Y}$ \\
2 & 2 & 0 & 29 & 0.835 & 0.132 & $\mathrm{Y}$ \\
3 -DP & 1 & 0 & 28 & 1 & 0.11 & $\mathrm{Y}$ \\
4 & 2 & 0 & 26 & 0.804 & 0.25 & $\mathrm{Y}$ \\
5 & 2 & 0 & 29 & 0.923 & 0.206 & $\mathrm{Y}$ \\
6 & 3 & 3.083 & 26 & 0.535 & 0.211 & $\mathrm{Y}$ \\
7 -ORL & 4 & 23.833 & 18 & 0.25 & 0.404 & $\mathrm{~N}$ \\
8 & 2 & 0 & 29 & 0.923 & 0.206 & $\mathrm{Y}$ \\
9 & 2 & 0.583 & 27 & 0.5 & 0.171 & $\mathrm{Y}$ \\
10 & 3 & 7 & 22 & 0.521 & 0.334 & $\mathrm{Y}$ \\
11 -HLM & 4 & 13.75 & 20 & 0.25 & 0.347 & $\mathrm{Y}$ \\
12 -HLM & 5 & 22.75 & 20 & 0.381 & 0.525 & $\mathrm{Y}$ \\
\hline
\end{tabular}

higher overall clustering coefficient of 0.705 , high transitivity of 25.97 percent, and highest average nodal degree from the sample cases of 4.308 (Table 12).

There exist two key players (HLMs) coordinating trafficking activities. While 10-HLM has a pivotal role with an actor coreness score of 0.541 , 1-HLM has a less prominent role being at the periphery (coreness 0.268 ) as plotted in Table 10. Information does not have to strictly flow through 10-HLM as indicated by the lower average geodesic distance (1.795), lower betweenness centrality (7.22 percent) and group centralization (36.11 percent), and the highest closeness centrality (57.33 percent) indicating a more efficiency oriented structure as depicted in Tables 11 and 12. As plotted in Fig. 10, bold ties between offenders indicate intense interactional relations despite no kinship tie within the network. However, the pre-existing friendship ties, as in all other sample networks, is an important characteristic of this network yielding more connectedness and an efficiency driven structure. Out of 13 offenders in this network, eight were captured, however five offenders, who were positioned on the periphery, were fugitives including one the key players, 1-HLM.

\section{Discussion}

This section discusses and relates the findings to prior research to address each of the developed hypothesis. It first addresses whether or not there exists a difference between DG and NT networks in their structure regarding the security-efficiency trade-off. This section also discusses overall samples-regardless of terrorist linkages-and network reliance on security and efficiency based on their structural characteristics and compares them with prior empirical research.

\section{Hypothsis-1: Illicit drug networks overall are denser and more degree centralized compared to narco-terror networks}

Centrality scores are plotted in Table 11 . Density and certain other cohesion and connectedness scores are reflected in Table 12. As previously noted, density scores tend to decrease as the size of a network increases (Everton, 2008: 98; Morselli et al., 2007: 148). As shown in Table 12 , average density score for NTNs $(0.25)$ is slightly lower than DGNs (0.29) depicting less ties in narco-terror networks. However, looking into individual networks from two camps does not yield systematic patterns showing distinction in densities. For instance, NT\#01 is denser than DG\#01, while both have a similar network size. NT\#04 and DG\#04 each with a size of 12 have almost the same level of density (0.24 and 0.26$)$ while DG\#02 with same size have higher density (0.37). On the other hand, NT\#02 and DG\#01 are particularly sparse (lowest density scores) with their star-like structure indicating centralized hubs with direct ties around incomplete cores on their edges. 


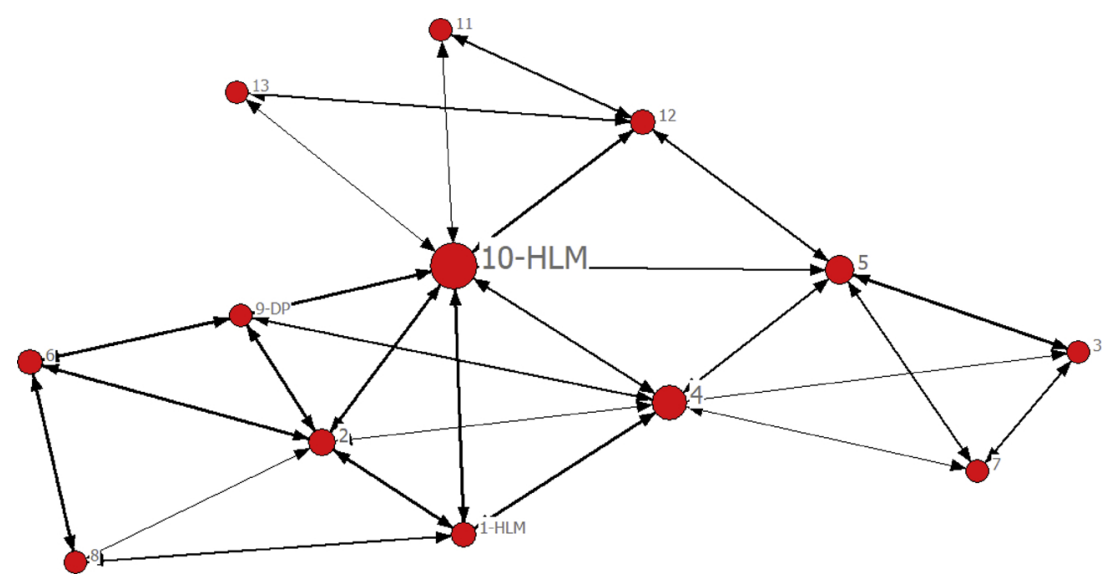

Fig. 10. Sociogram for the Network DG\#05.

Table 10

Network DG\#05 - Actor-by-actor power and centrality scores.

\begin{tabular}{lllllll}
\hline Node & Degree & Between & Close & Constraint & Coreness & Arrest \\
\hline 1 -HLM & 4 & 3.233 & 20 & 0.452 & 0.268 & $\mathrm{~N}$ \\
2 & 6 & 5.233 & 18 & 0.447 & 0.373 & $\mathrm{Y}$ \\
3 & 3 & 0 & 25 & 0.704 & 0.137 & $\mathrm{Y}$ \\
4 & 7 & 14.067 & 17 & 0.351 & 0.404 & $\mathrm{Y}$ \\
5 & 5 & 6.667 & 20 & 0.428 & 0.254 & $\mathrm{Y}$ \\
6 & 4 & 2.033 & 22 & 0.492 & 0.244 & $\mathrm{Y}$ \\
7 & 3 & 0 & 25 & 0.704 & 0.137 & $\mathrm{Y}$ \\
8 & 3 & 0.333 & 27 & 0.552 & 0.153 & $\mathrm{~N}$ \\
$9-\mathrm{DP}$ & 4 & 0.833 & 20 & 0.497 & 0.288 & $\mathrm{Y}$ \\
$10-\mathrm{HLM}$ & 9 & 26.767 & 15 & 0.286 & 0.541 & $\mathrm{Y}$ \\
11 & 2 & 0 & 25 & 0.699 & 0.124 & $\mathrm{~N}$ \\
12 & 4 & 2.833 & 21 & 0.534 & 0.183 & $\mathrm{~N}$ \\
13 & 2 & 0 & 25 & 0.699 & 0.124 & $\mathrm{~N}$ \\
\hline
\end{tabular}

Other than a tiny difference (0.04) in average densities, sample networks, regardless of whether DG or NT, do not reflect systematic difference in density as depicted in Table 12.

In general, except NT\#02 and DG\#01, sample networks reflect denser and sparser structures when compared to covert networks analyzed in the literature. For instance, Baker and Faulkner (1993: 851) analyzed conspiracy networks and found a density of 0.35 for a network with a size of 24 and a density of 0.32 for a network with a size of 21 indicating denser structures than Turkish counterparts with similar size, NT\#01 (size 21, density $=0.20$ ) and DG\#01 (size 23, density $=0.14$ ). However, these two sample networks seem to be denser (smaller network size should be taken into consideration) when compared to a drug network comprised of 38 members (network's core) yielding 0.12 density as reported by Natarajan (2006: 183) and when compared to
American based Hezbollah trade diversion network comprised of 34 with 2.202 density as reported by Belli et al. (2015: 270). Yet, when compared to $9 / 11$ attack network comprised of 16 members with density of 16, Turkish sample networks (both, narco-terror and illicit drug) with even smaller size, e.g., NT\#03(14), NT\#04(12), NT\#05(10), DG\#02(12), DG\#03(11), DG\#04(12), DG\#05(13) indicate denser structures ranging from densities of 0.24 to 0.38 (except the NT\#02 with similar size of 17 indicating sparser structure with 0.13 density). On the other hand, Bright et al. (2012: 163) reported that densities of criminal networks in illicit markets including drug and price fixing groups range between 0.05 to 0.25 . Given these, most sample networks, both NTs and DGs seem to have denser structures given their size and other cohesion and connectedness metrics (elaborated under $\mathrm{H}_{2}$ ). As noted in the literature review, there is no accepted threshold value to deem a covert network's sparseness or denseness (Oliver et al., 2014: 8). So, Turkish drug networks either with or without a terrorist nexus prioritize efficiency with more incidence of relations for effective communication and coordination.

On the other hand, Milward and Raab (2006: 344-45) argue that networks with shared values and pre-existing ties tend to have higher density, while some claim that specific attributes of members affect density: for instance, more skilled members leads to less dense structures (e.g., Helfstein and Wright (2011, 806). In light of these, higher densities of sample networks can be explained with strong pre-existing trust-ties, both kinship and friendship, as reflected in Table 13 (elaborated under $\mathrm{H}_{4}$ ).

With regard to centrality and centralization, results are mixed in the literature. While Natarajan (2006) and Klerks (2001) find illicit groups to be degree decentralized for security concerns, Baker and Faulkner (1993) find three conspiracy networks to be reasonably centralized (60.8 percent) and claim the opposite. Considering the average scores,

Table 11

Networks NT and DG - Average centrality and group centralization results.

\begin{tabular}{|c|c|c|c|c|c|c|c|c|c|}
\hline \#CASE & Size & Terr. Ratio \% & Density 0-1 & Degree \% & Closeness \% & Betweenness \% & Degree & Closeness & Betweenness \\
\hline NT01 & 21 & 9.52 & 0.20 & 20.47 & 41.51 & 77.19 & 21.58 & 27.03 & 23.19 \\
\hline NT02 & 17 & 11.76 & 0.13 & 13.23 & 35.55 & 12.94 & 27.50 & 43.07 & 58.85 \\
\hline NT03 & 14 & 14.29 & 0.29 & 29.67 & 51.96 & 73.26 & 46.15 & 48.09 & 31.69 \\
\hline NT04 & 12 & 58.33 & 0.24 & 25.75 & 49.50 & 12.44 & 34.55 & 53.31 & 34.05 \\
\hline NT05 & 10 & 50 & 0.38 & 37.77 & 55.03 & 9.44 & 50 & 53.31 & 40.95 \\
\hline Average & 14.8 & 28.77 & 24.8 & 25.37 & 46.71 & 37.05 & 35.96 & 44.96 & 34.05 \\
\hline DG01 & 23 & 0 & 0.14 & 14.62 & 43.34 & 65.97 & 38.74 & 45.74 & 49.43 \\
\hline DG02 & 12 & 0 & 0.37 & 36.36 & 56.9 & 8.33 & 54.55 & 63.49 & 44.93 \\
\hline DG03 & 11 & 0 & 0.33 & 32.72 & 54.91 & 9.89 & 57.78 & 66 & 53.89 \\
\hline DG04 & 12 & 0 & 0.26 & 25.75 & 45.79 & 12.42 & 23.64 & 35.09 & 33.72 \\
\hline DG05 & 13 & 0 & 0.36 & 35.89 & 57.33 & 7.22 & 46.21 & 51.33 & 36.11 \\
\hline Average & 14.2 & 0 & 0.29 & 32.68 & 53.73 & 9.46 & 45.54 & 53.97 & 42.16 \\
\hline
\end{tabular}

All centrality measures are normalized to 0-100 percentage. 
Table 12

Networks NT and DG - Cohesion and connectedness scores.

\begin{tabular}{|c|c|c|c|c|c|c|c|c|}
\hline \#CASE & Size & $\begin{array}{l}\text { Terr Ratio } \\
\%\end{array}$ & Density $0-1$ & $\begin{array}{l}\text { Overall Clustering } \\
\text { Coefficient }\end{array}$ & $\begin{array}{l}\text { Average Geo. Distance } \\
\text { (Path Length) }\end{array}$ & $\begin{array}{l}\text { Network Transitivity \% } \\
\text { (Adjacency) }\end{array}$ & $\begin{array}{l}\text { Average Nodal } \\
\text { Degree }\end{array}$ & $\begin{array}{l}\text { Core-Peripheriness } \\
\text { (Correlation) }\end{array}$ \\
\hline NT01 & 21 & 9.52 & 0.20 & 0.64 & 2.47 & 22.61 & 4.09 & 0.4 \\
\hline NT02 & 17 & 11.76 & 0.13 & 0.17 & 2.94 & 2.632 & 2.11 & 0.52 \\
\hline NT03 & 14 & 14.29 & 0.29 & 0.71 & 1.88 & 21.25 & 3.85 & 0.56 \\
\hline NT04 & 12 & 58.33 & 0.24 & 0.63 & 2.1 & 22.64 & 2.66 & 0.54 \\
\hline NT05 & 10 & 50 & 0.38 & 0.78 & 1.76 & 25.64 & 3.40 & 0.66 \\
\hline Average & 14.8 & 28.8 & 24.8 & 0.59 & 2.23 & 22.64 & 3.22 & 0.46 \\
\hline DG01 & 23 & 0 & 0.14 & 0.41 & 2.38 & 6.74 & 3 & 0.47 \\
\hline DG02 & 12 & 0 & 0.37 & 0.71 & 1.83 & 27.69 & 4 & 0.64 \\
\hline DG03 & 11 & 0 & 0.33 & 0.79 & 1.89 & 21.95 & 3.27 & 0.62 \\
\hline DG04 & 12 & 0 & 0.26 & 0.46 & 2.24 & 9.09 & 2.83 & 0.34 \\
\hline DG05 & 13 & 0 & 0.36 & 0.71 & 1.79 & 25.97 & 4.30 & 0.51 \\
\hline Average & 14.2 & 0 & 0.29 & 0.62 & 2.03 & 18.28 & 3.27 & 0.52 \\
\hline
\end{tabular}

Table 13

Networks NT and DG - Pre-existing ties and Density.

\begin{tabular}{lllllll}
\hline Case \# & Size & \#Kinship & \%Kinship & \#Peer & \%Peer & Density \\
\hline NT01 & 21 & 7 & 33.3 & 0 & 0 & 0.20 \\
NT02 & 17 & 6 & 35.29 & 14 & 82.35 & 0.13 \\
NT03 & 14 & 3 & 21.42 & 11 & 78.6 & 0.29 \\
NT04 & 12 & 4 & 33.3 & 11 & 91.6 & 0.24 \\
NT05 & 10 & 3 & 30 & 9 & 90 & 0.38 \\
Average & 15.5 & 6.5 & 33.3 & 8.5 & 56.2 & 0.24 \\
DG01 & 23 & 4 & 17.39 & 20 & 86.96 & 0.14 \\
DG02 & 12 & 2 & 16.66 & 11 & 91.66 & 0.37 \\
DG03 & 11 & 3 & 27.27 & 10 & 90.9 & 0.33 \\
DG04 & 12 & 4 & 33.33 & 11 & 91.66 & 0.26 \\
DG05 & 13 & 0 & 0 & 12 & 92.3 & 0.36 \\
Average & 14.2 & 2.25 & 19.31 & 11 & 91.63 & 0.29 \\
\hline
\end{tabular}

NTNs are found to be slightly less centralized than DGNs in degree and closeness, meaning that members in DGNs have more direct and closer ties while, given the betweenness centrality, NTNs reflect more intermediation/brokerage in their structure. However, analysis of individual networks' centrality—similar to the density—reveals no systematic difference between the two camps. Both NTNs and DGNs have pivotal actors constituting central cores with independent ties (e.g., NT\#02 and DG\#01) or with dense clusters and complete edges around them (e.g., NT\#03, DG\#02, DG\#05) and/or with strict gateways brokering separate clusters (e.g., NT\#04, DG\#03). Similar to the literature, analyses of centrality of sample networks of NT and DG networks reveal mixed results as depicted in Table 11. They reflect lower and higher results for different centrality and centralization metrics indicating different structural implications. The cases NT\#02 and DG\#01, for instance, have lowest density and degree centrality for security (see e.g., Lindelauf et al., 2009), however, they indicate central actors (hubs) with strict control and coordination of the flow of information and resources for efficient communication which causes the security vulnerability of structural holes (see e.g., Ender and Su 2007) with the highest average betweenness centralization (58.85 and 49.43 percent) as depicted in Table 11. Yet, NT\#01, NT\#03, and DG\#01 are featured with high intermediation, others are lower in strict brokerage. On the other hand, members are closer in DG\#05, DG\#02, NT\#02, NT\#03 and NT\#05 compared to other cases from two camps.

Sample NTNs and DGNs analyzed in this study, in general, does not reveal a clear difference in terms of cohesiveness and centralization. Therefore, despite terrorist members being in key roles and central positions, results do not support the assertion that narco-terror networks-having terrorist nature-are more security driven with lower density and less degree centralization. Overall network structures reflect centralized and decentralized characteristics in different metrics reflecting different organizational and social structural inferences on security and efficiency concerns as elaborated in the following hypotheses (e.g., clusters, core-peripheriness, pre-existing ties, positions of key players).

Hypothsis-2: Illicit drug networks are more clustered into dense sub-groups and have shorter (path) distance compared to narco-terror networks

Overall, the average scores indicate that DGNs are slightly more clustered (0.62) and have slightly shorter paths (2.03) than NTNs (clustering coefficient of 0.59 , and average geodesic distance of 2.23). All 10 sample networks, however, have almost same average nodal degree (3.27 and 3.22 respectively). Yet, individual networks reflect high clustering and very short path lengths for networks from both categories. Networks from narco-terror (NT\#03 N\#05) and illicit drug (DG\#02 DG\#03 DG\#05) camps both have high coefficients (over 0.70) of clustering with short path lengths (below 1.90), while certain other cases from each camp have lower clustering and a longer path length as plotted in Table 12. NT\#02 has the lowest clustering (0.17) and longer path length (2.94) and the lowest transitivity indicating the least complete edges which reflects central hubs relying strictly on authoritative key players' strict control constituting structural holes.

Compared to the September 11 hijack cell with an average path length of 4.75 with 19 hijackers (Krebs, 2002), sample networks of both NTs and DGs indicate shorter paths between offenders in general. Again, sample networks reflect different structures in terms of security and efficiency. Networks DG\#01 and NT\#02 have highly central actors with pivotal roles and direct ties to unconnected actors controlling and coordinating the flow of communication and resources. This structure is suggested to be more optimal (in security) by Lindelauf et al. (2009), in that covert networks are to be more degree centralized hub (star like), have shorter path lengths (sparse structure) between members, when operating in hostile environment-in their initial stage of operation-as typically reflected in DG\#01 and NT\#02. However, despite the low incidence and less direct ties among members, such a structure risks the complete termination of the network when these central hubs are compromised (Enders and Su, 2007). Network DG\#04, on the other hand, seems to be more security driven with a flattened (snake-like) structure lacking a clear core evident in low scores of network centralization (23.64 percent) and core-peripheriness (0.341) as depicted in Tables 11 and 12.

In light of these results, one can hardly argue for a systematic structural difference between NT and DG networks in terms of paths between members and clustering into sub-groups. The majority of the sample networks support the existence of microstructures (dense clusters and short path lengths) in covert networks as argued by Morselli et al (2007); Oliver et al. (2014) and Crossley et al. (2012). Results indicate that all sample networks, whether NTNs or DGNs, tend to reflect small and relatively denser clusters in their cores and peripheries and short distance among members reflecting more reliance on efficiency in communication. 
Hypothsis-3: Illicit drug networks have more coreness reflecting more coreperiphery structures compared to narco-terror networks

Overall, when compared to average core-peripheriness of NTNs (correlation of 0.46 ), the average scores of core-peripheriness indicate that DGNs (correlation of 0.52) have slightly more ties among central actors while peripheral actors tend to have weaker ties with one another. While this tiny difference can be deemed in supportive of the aforementioned assertion, we cannot say that NTNs lack a core. Analyzing the visual representation of networks, sociograms, indicate that most sample networks, including NTNs, indicate existence of cores with dense structures (e.g., NT\#03, NT\#05 and DG\#02, DG\#05) or with more direct ties to central cores (e.g., NT\#02 and DG\#01). Therefore, sample networks, regardless of having terrorist members, indicate that there exist core(s) in sample networks. Yet, as indicated in Table 12, except for three cases, core-peripheriness (correlation) scores are over 0.5. For NT\#01, since the network yields a polycentric structure, the core-peripheriness reflects lower score for the entire network, however each of the sub-groups reflect dense cores connected to one another via certain key players with high control and brokerage role (for the importance of weak ties see Granovetter, 1973).

In the literature, existence of core-periphery (polycentric) structures are denoted as an emerging feature of covert networks (Oliver et al. 2012: 10). In that, contrary to Morselli et al. (2007: 152-53) core structures are found even in terrorist networks comprised of specialized members as argued by Raab and Milward (2003: 413-9) and Helfstein and Wright (2011, 805-07). More over, Belli et al. (2015: 269) reports that American based Hezbollah trade diversion network (nature of the objective s criminal) with 34 members indicated 0.679 of degree centralization, revolving around a ringleader for efficient communication.

In the end, there is no clear difference between NT and DG networks in that most sample networks, regardless of terrorist linkages, reflect the existence of network cores to have more efficient communication and control. In that, offenders in sample networks, similar to the trade diversion network of the Hezbollah based in the US, were willing to sacrifice some degree of security in favor of a more efficient operation with shorter paths between core and periphery (Belli et al., 2015: 276). Thus, results do not support the above hypothesis in that narco-terror networks (having terrorist nexus) have less coreness. However, it should be noted that while Morselli et al. (2007) claim that criminal covert networks are built upon a core while terrorist networks lack a core, they also claim that nature of the objective determines the structural characteristics of a covert networks. From this perspective, results support their claim in that despite terrorists being key players and taking central positions in narco-terror networks, networks have cores in their structure. Hence, having terrorists in dominant roles and positions did not influence methods since networks are still materially driven in their objective.

Hypothsis-4: Key players in drug networks tend to be positioned more in the networks' core for more efficient control and coordination while key players and terrorists in Narco-terror networks are positioned more in periphery structures to maintain more security.

As delineated through actor-by-actor coreness scores and plotted sociograms in the discussion of each case, results indicated that key players (ORLs and HLMs) in NT networks are positioned in the central (controlling a hub) and/or critical (gateway with high brokerage role) positions. More importantly, most of the key players in NTNs are terrorists (members with strong linkages to the PKK). The situation is not different for the DGNs. Key players having strategic roles (initiating the trafficking crime, exerting authority and control, and owning the crime assets) are mostly positioned in the cores of the sample networks to control and coordinate the activity efficiently.

However, there also exist key players positioned at the peripheries of these networks with multiple key players in each sample from both network types. While NT\#01, NT\#02, and DG\#01 have key players in critical positions (act as central core or gateway), DG\#02, DG\#03, and
DG\#05 have key players also in their peripheries. For network DG\#02 for instance, one of the organizational leaders is found to be less prominent with only one tie to the network's core refraining from the security vulnerability of detection while other key players are positioned in network's core. Among all the sample networks, narco networks of NT\#03, NT\#04, and NT\#05 have key players that tend to be more in the periphery. However, in two networks, NT\#04 and NT\#05, terrorists are positioned in the pivotal (central) and critical (gateway) positions as opposed to the expectation that they would be positioned in the periphery to maintain more security with less visibility.

In the end, results do not support the above claim that terrorists and key players are positioned in the periphery to protect against security vulnerabilities unlike in illicit drug networks. Likewise, results for all samples, with or without a terrorist nexus, do not fully support the aforementioned assertion made by Baker and Faulkner (1993) that while most of the leaders (except one) are positioned in the core of sample networks, certain high level managers are positioned in the peripheries-although with high degree (direct ties) scores. The general argument in the literature indicates that leaders in a covert network, just like the dilemma for the overall network, want to exert their authority by controlling and coordinating activity through a central role on one hand, and on the other, as Crossley et al. (2012: 2) suggested, avoid a high degree to maintain more secrecy/security; this may lead to having a more betweenness centrality as evident, for instance, in the case NT\#01. For efficiency reasons, however, sample Turkish criminal networks, regardless of having terrorist members, seem to have key players with more central and pivotal positions with power, authority, and brokerage (degree and betweenness) indicating the reliance more on efficiency in general. This finding is similar to the results from the study of Islamic extremist (American-based Hezbollah) network involved in trade diversion (cigarettes), in which ringleader (key player) yields a position to oversee the overall functioning of network and directly linked to several network members for the sake of operational efficiency (Belli et al., 2015: 276).

When considering the arrests and fugitives, results revealed that-except certain specific offenders in NT\#03 NT\#05 DG\#02 and DG\#04-all fugitives from all sample networks were on the peripheries of these covert networks with lesser degree centrality. This concurs Morselli's (2010:382) study of a criminal organization (the Quebec Hells Angels) in showing how differential positions in the network influence the (judicial outcomes) capture and arrests. In that, there is strong correlation between high degree centrality and possibility for the actor to get caught and arrested.

Hypothsis-5: Narco terror and illicit drug networks have pre-existing social structures (kinship and peer)

Results revealed that while kinship structure is more prominent in NTs, peer and friendship structure is greater in DGs as depicted in Table 13. Sample networks, overall, have high levels of pre-existing ties of both kinship and friendship. Except network DG\#05, all sample cases have kinship structures in varying degree. All networks except NT\#01 have strong peer and friendship ties as denoted with high percentages in Table 13. Kinship structures, as plotted (denoted with yellow nodes) in sociograms, exist in both central cores (e.g., NT\#01, NT\#04, DG\#01, DG\#03) and dense clusters and cliques in the peripheries (e.g., NT\#02, NT\#03, NT\#05, DG\#02).

The overall results support claims made by Erickson (1981) and many others in the literature. Pre-existing ties and social bonds known as 'trust ties' are among important characteristics of covert networks (Raab and Milward, 2003; Milward and Raab, 2006 :349). Trust-ties reduce vulnerability and increase resilience (Oliver et al., 2014: 4) through internal policing reinforcement of in-group solidarity (Milward and Raab, 2006) and facilitate self-sacrifice and group solidarity (Crossley et al., 2012: 3). Thus, as Erickson (2001) and Sageman (2011) argued, pre-existing contacts are important for the growth of networks 
through their members making social ties and trust-based relations important for social structures of dark networks.

From a social network perspective, relational ties between offenders can exert a robust influence on the collective behavior of the group. This can occur particularly in areas where family ties and bonds are culturally strong as is the case of Columbian drug cartels and insurgent groups (e.g., FARC) as well as the PKK and Kurdish ethnographical ties in Turkey's southeastern region which is marked with feudal social structures with strong familial and blood ties (Ünal and Ünal, 2017). In short, social bonds matter in how these covert networks are structured, operate, and grow. The use of social bonds in pre-existing ties constitutes the ties of trust which contribute to effectiveness and security both internally and externally.

\section{Hypothsis-6: More endured covert networks with reputation are more security driven}

The overall picture drawn from offender statements indicates that all sample networks analyzed in this study, regardless of being NT or DG organizations, reflect small, flexible and fluid enterprises that do not reveal endurance, institutionalization, or stability.

The literature on drug networks in general and Turkish drug networks in particular supports this claim. As known, illicit drug networks, like other criminal and terrorist organizations, do not reflect a standard character varying in many respects (e.g., scale, form, structure, modus operandi) based on factors originating from geo-spatial conditions in which they operate (e.g., type of illicit drug to be trafficked, level of stability, border security and nature of terrain in their habitat) (Raab and Milward, 2003; Makarenko, 2004; Howard and Traughber 2006; Hutchinson and O'Malley, 2007). For instance, these organizations expand, enlarge or shrink their activities and shape their organizational structures based on the aggressiveness of the law enforcement agencies, availability of regions to cultivate, possible means for production and transportation of drugs. Colombian drug cartels (most of them linked to FARC) or Afghan opium producers (most of them linked to Taliban or Al-Qaida) reflect large organizations with some control over some regions in the country (Makarenko, 2004; Howard and Traughber 2006; Hutchinson and O'Malley, 2007). However, drug networks in Europe, due to environmental conditions, are small in size and more underground in structure reflecting their more decentralized nature (Makarenko and Mesquita, 2014). Drug networks also vary based on their focus within the drug trafficking cycle (pre-production, cultivation, production, storage, distribution) which also reflects change in the level of market focus (Levitt and Venkatesh, 2000). In sum, the characteristics and structure of drug networks change according to the country with their social, economic, and political environment reflecting different strategic needs and contextual contingencies (Raab and Milward, 2003: 430). Given this, Turkish drug networks, while changing in time like others, reflect small-size, pragmatic enterprises rather than reflecting endurance and reputation. They are featured with kinship and peer structures similar to their Russian and Caucasian counterparts. Also, like Afghan and Tajik networks, Turkish networks have certain geographical advantages in supply and transportation through the Southeastern and Eastern regions of Turkey (Turhal, 2015). Most of the drug networks are involved in trafficking of illicit drugs which does not include pre-production, cultivation, and production processes. In the end, Turkish drug networks are profit-oriented, small entities reflecting rational, pragmatic, flexible, and fluid characteristics in which their structure and function are driven mostly by material incentives rather than reputation and endurance.

\section{Conclusion}

This study empirically analyzed the overall nature and characteristics of ten criminal networks in the Turkish context, five illicit drug networks and five (PKK affiliated) narco-terror networks. The five sample narco-terror networks-that are compared with the illicit drug networks in this study-are not purely ideology-driven networks, rather they are narco-terror networks with PKK militants/terrorists operating in them to drive financial gains. This study examined these networks' structural characteristics regarding the security-efficiency trade-off and analyzed whether or not having terrorist linkages (crime terror-nexus) has any structural influence in prioritizing between security and efficiency.

Results indicated that slight differences exist in average scores of certain cohesion, connectedness and centrality measures, however not to an extent that would imply systematic and significant difference. In that narco-terror networks' average scores show slightly sparser structure with slightly lesser direct ties (centrality) and less centralization around pivotal actors than illicit drug networks. In line with this, narcoterror networks' average scores show slightly less clustering into dense subgroups with slightly longer (average geodesic distance) paths between members, which technically indicates more reliance on security than efficiency when compared to illicit drug networks. However, the magnitude of difference in average scores of sample networks are insignificant, and individual networks from both network types reveal mixed results (higher and lower densities and centralizations). These results yield no clear structural differences between the network types.

Overall, most of the sample networks, regardless of being narcoterror or mere illicit drug networks, reflect dense clusters attached to core and peripheries, while certain others reveal sparse structures with less individual ties around central hubs (causing security vulnerability-structural holes-of complete termination when hubs are compromised). Yet, while certain samples reflect clear cores with key players positioned in centers, certain others yield a polycentric nature with key players in high brokerage/intermediation roles or, in certain cases, key players positioned in peripheries. Despite narco-terror networks having more kinship ties, illicit drug networks revealed more peer and friendship ties. As a common feature, all samples contained strong pre-exiting trust ties.

In the aforementioned regards, most of the sample cases, as criminal (illicit drug and narco terror) networks, are found to be more efficiency driven compared to criminal networks analyzed in the literature. When compared to prior research, most sample networks-in general-have higher density (more ties) and shorter distance (path) between members to better control and coordinate the trafficking activity through more effective communication. Sample networks are clustered into denser subgroups attached to network cores and, in certain instances, to the peripheries, reflecting microstructures of tightly-knit clusters and cliques that makes them more transitive (complete triads) in communication structures. Certain sample networks, however, are sparser with central hubs having individual ties with incomplete edges. Analyses of both drug and narco-terror networks revealed that most networks have core(s) to have more efficient communication as well as more effective control and coordination. Key players are mostly found to act in pivotal positions to exert power, authority, and brokerage for more convenient control and coordination, while certain key players-leaders and managers-were positioned at the periphery. This risks detection and thus increases the susceptibility of disruption through more visibility and structural holes due to highly centralized pivotal positions (high damage when compromised).

All sample networks, both drug and narco-terror ones, revealed strong pre-existing social bonds and reflected kinship and peer structures reinforcing internal security (trust-ties against defection and infiltration), growth (recruitment), and resilience (in-group solidarity, self-sacrifice) as well as efficiency through denser clusters within and outward networks' cores. Likewise, as profit-oriented small entities reflecting rational, flexible, and fluid characteristics, the structure and function of drug networks are seen to be driven mostly by material incentives.

In the end, most of the sample networks from both camps, illicit drug and narco-terror, are more efficiency driven than security, while 
only three of the sample networks (NT\#02, DG\#01, and DG\#04) seem to reflect certain security driven structures.

Despite terrorist (PKK) members being engaged in narco-terror networks in powerful (central and intermediate roles) positions, results did not bring significant structural difference from their mere illicit drug counterparts. Therefore, the nature of objective dominates the structural characteristics towards the security-efficiency dilemma and, in this sense, the network is designed for greater efficiency while striving for as much security as possible. Given this, this study asserts that a covert network's objective determines the incidence of action. This then designs network structures by bringing members together to accomplish the predetermined objective; criminal networks, illicit drug organizations as well as narco-terror networks in Turkish context, are driven by material incentives and thus rely more on efficiency.

As a result, having PKK terrorists in narco-terror networks does not make systematic and structural difference for criminal activity: illicit drug trafficking driven by material incentive. More specifically, despite slight differences in average scores, there is no clear and systematic distinctive nature in structural characteristics between illicit drug networks and their narco-terror counterparts in the Turkish context. Most of the sample networks, whether illicit drug or narco-terror, tend to rely more on efficiency than security when compared to criminal cases analyzed in the literature. Yet, sample networks are small, flexible, and fluid enterprises that are mostly structured to accomplish their profitbased criminal aims in which they strive to balance the dilemma between efficiency and security in specific ways: while being more efficiency driven-given their structural characteristics-they try to compensate for security vulnerabilities through individual social characteristics-pre existing trust ties, which brings important policy implications that social bonds and ties (friendship and relatives) for narco-terror and illicit drug networks in Turkey may provide an important tool for law enforcement agencies.

\section{References}

Asal, V., Milward, H.B., Schoon, E.W., 2015. When terrorists go bad: analyzing terrorist organizations' involvement in drug smuggling. Int. Stud. Q. 59 (1), 112-123.

Baker, W.E., Faulkner, R.R., 1993. The social organization of conspiracy: illegal networks in the heavy electrical equipment industry. Am. Sociol. Rev. 837-860.

Belli, R., Freilich, J.D., Chermak, S.M., Boyd, K.A., 2015. Exploring the crime-terror nexus in the United States: a social network analysis of a hezbollah network involved in trade diversion. Dyn. Asymm. Confl. 8 (3), 263-281.

Björnehed, E., 2004. Narco-terrorism: The merger of the war on drugs and the war on terror. Global Crime 6 (3-4), 305-324.

Borgatti, S.P., Everett, M.G., 2000. Models of core/periphery structures. Soc. Networks 21 (4), 375-395.

Bright, D.A., Hughes, C.E., Chalmers, J., 2012. Illuminating dark networks: a social network analysis of an Australian drug trafficking syndicate. Crime Law Soc. Change 57 (2), 151-176.

Burcher, M., Whelan, C., 2015. Social network analysis and small group 'dark'networks: an analysis of the London bombers and the problem of 'fuzzy'boundaries. Global Crime 16 (2), 104-122.

Crossley, N., Edwards, G., Harries, E., Stevenson, R., 2012. Covert social movement networks and the secrecy-efficiency trade off: The case of the UK suffragettes (1906-1914). Soc. Networks 34 (4), 634-644.

Duxbury, S.W., Haynie, D.L., 2018. Building them up, breaking them down: topology, vendor selection patterns, and a digital drug market's robustness to disruption. Soc. Networks 52, 238-250.

Edwards, G., 2014. Infectious innovations? The diffusion of tactical innovation in social movement networks, the case of suffragette militancy. Soc. Mov. Stud. 13 (1), 48-69.

Enders, W., Su, X., 2007. Rational terrorists and optimal network structure. J. Conflict Resolut. 51 (1), 33-57.

Erickson, B.H., 1981. Secret societies and social structure. Soc. Forces 60 (1), 188-210.

Everton, S.S., 2008. Tracking, Destabilizing and Disrupting Dark Networks With Social Networks Analysis.

Freeman, L.C., Roeder, D., Mulholland, R.R., 1979. Centrality in social networks: II. Experimental results. Soc. Networks 2 (2), 119-141.
Granovetter, M.S., 1973. The strength of weak ties. Am. J. Sociol. 78 (6), 1360-1380.

Hanneman, R., Riddle, M., 2005. Introduction to Social Network Analysis. Riverside CA. University of California.

Helfstein, S., Wright, D., 2011. Covert or convenient? Evolution of terror attack networks. J. Conflict Resolut. 55 (5), 785-813.

Howard, R.D., Traughber, C.M., 2008. 27 The "New Silk Road" of Terrorism and Organized Crime: The Key to Countering the Terror-Crime Nexus.

Hudson, R., Berry, L., Curtis, G.E., Nina, A.K., 2002. A global overview of narcotics funded terrorist and other extremist groups. May. Library of Congress.

Hutchinson, S., O'malley, P., 2007. A crime-terror nexus? Thinking on some of the links between terrorism and criminality. Stud. Confl. Terrorism 30 (12), 1095-1107.

Jordan, J., Mañas, F.M., Horsburgh, N., 2008. Strengths and weaknesses of grassroot jihadist networks: The Madrid bombings. Stud. Confl. Terrorism 31 (1), 17-39.

Klerks, P., 2001. The network paradigm applied to criminal organizations. Theoretical nitpicking or a relevant doctrine for investigators? Recent developments in the Netherlands. Connections 24 (3), 53-65.

Knoke, D., Yang, S., 2008. Social Network Analysis 154 Sage.

Koschade, S., 2006. A social network analysis of Jemaah Islamiyah: The applications to counterterrorism and intelligence. Stud. Confl. Terrorism 29 (6), 559-575.

Krebs, V.E., 2002. Mapping networks of terrorist cells. Connections 24 (3), 43-52.

Levitt, S.D., Venkatesh, S.A., 2000. An economic analysis of a drug-selling gang's finances. Q. J. Econ. 115 (3), 755-789.

Lindelauf, R., Borm, P., Hamers, H., 2009. The influence of secrecy on the communication structure of covert networks. Soc. Networks 31 (2), 126-137.

Makarenko, T., 2004. The crime-terror continuum: tracing the interplay between transnational organised crime and terrorism. Global Crime 6 (1), 129-145.

Makarenko, T., Mesquita, M., 2014. Categorising the crime-terror nexus in the European Union. Global Crime 15 (3-4), 259-274.

Mayntz, R., 2004. Organizational Forms of Terrorism: Hierarchy, Network, or a Type Sui Generis? No. 04/4). MPIfG Discussion Paper.

McGloin, J.M., Kirk, D.S., 2010. An overview of social network analysis. J. Crim. Justice Educ. 21 (2), 169-181.

Milward, H.B., Raab, J., 2006. Dark networks as organizational problems: elements of a theory. Int. Public. Manage. J. 9 (3), 333.

Morselli, C., 2009. Inside Criminal Networks 8 Springer, New York.

Morselli, C., 2010. Assessing vulnerable and strategic positions in a criminal network. J. Contemp. Crim Justice 26 (4), 382-392.

Morselli, C., Giguère, C., Petit, K., 2007. The efficiency/security trade-off in criminal networks. Soc. Networks 29 (1), 143-153.

Natarajan, M., 2000. Understanding the structure of a drug trafficking organization: a conversational analysis. Crime. Prev. Stud. 11, 273-298.

Natarajan, M., 2006. Understanding the structure of a large heroin distribution network: A quantitative analysis of qualitative data. J. Quant. Criminol. 22 (2), 171-192.

Oliver, K., Crossley, N., Edwards, G., Koskinen, J., Everett, M., Broccatelli, C., 2014 Covert Networks: Structures, Processes and Types. Unpublished manuscript. University of Manchester, Manchester, UK.

Paoli, L., 2001. Drug trafficking in Russia: a form of organized crime? J. Drug Issues 31 (4), 1007-1037.

Raab, J., Milward, H.B., 2003. Dark networks as problems. J. Public. Adm. Res. Theory 13 (4), 413-439.

Rodriguez, J., 2009. Weakness and strengths of terrorist networks: The Madrid March 11 attacks. Annual Meeting of the American Sociological Association. Available at http://www. allacademic. com/meta/p243052 index. html.

Sageman, M., 2011. Leaderless Jihad: Terror Networks in the Twenty-First Century. University of Pennsylvania Press.

Sparrow, M.K., 1991. The application of network analysis to criminal intelligence: an assessment of the prospects. Soc. Networks 13 (3), 251-274.

Turhal, T., 2015. Organizational Structure of PKK and Non-PKK-Linked Turkish Drug Trafficking Organizations: The Influence of Social Bonds. Doctoral dissertation. .

Ünal, M.C., 2012. Counterterrorism in Turkey: Policy Choices and Policy Effects Toward the Kurdistan Workers' Party (PKK), the Series of the "Democracy and Extremism. Routledge, London, UK.

Ünal, M.C., 2014. Strategist or pragmatist: a challenging look at ocalan's retrospective classification and definition of PKK's strategic periods between 1973 and 2012. Terrorism Political Violence 26 (3), 419-448.

Ünal, M.C., 2016. Terrorist or insurgency: A conceptual analysis. Crime Law Soc. Change 65 (6), 460-494.

Ünal, M.C., Ünal, T., 2017. Recruitment or enlistment? Individual integration into the Turkish hezbollah. Turk. Stud, 1-36.

Van der Hulst, R.C., 2009. Introduction to social network analysis (SNA) as an investigative tool. Trends Organ. Crime 12 (2), 101-121.

Wasserman, S., Faust, K., 1994. Social Network Analysis: Methods and Applications 8 Cambridge university press.

Watts, D.J., 1999. Networks, dynamics, and the small-world phenomenon. Am. J. Sociol. 105 (2), 493-527.

Zech, S.T., Gabbay, M., 2016. Social network analysis in the study of terrorism and insurgency: from organization to politics. Int. Stud. Rev. 18 (2), 214-243. 